\title{
Stress response in Entamoeba histolytica is associated with robust processing of tRNA to tRNA-halves
}

Manu Sharma ${ }^{1}$, Hanbang Zhang $^{1}$, Gretchen Ehrenkaufer ${ }^{1}$, and Upinder Singh ${ }^{1,2, \#}$

1. Division of Infectious Diseases, Stanford University School of Medicine.

2. Department of Microbiology and Immunology, Stanford University School of Medicine

\# Corresponding author: usingh@stanford.edu; Phone 650-723-4045; Address: Division of

Infectious Diseases, Grant Building S-143, 300 Pasteur Drive, Stanford University School of Medicine, Stanford, CA, 94305.

Running title: tRNA-derived fragments in Entamoeba histolytica

Keywords: Entamoeba, tRNA-derived fragments, tRNA halves, encystation 


\begin{abstract}
$\underline{\text { Abstract }}$
tRNA-derived fragments have been reported in many different organisms and have diverse cellular roles such as regulating gene expression, inhibiting protein translation, silencing transposable elements and modulating cell proliferation. In particular tRNA halves, a class of tRNA fragments produced by the cleavage of tRNAs in the anti-codon loop, have been widely reported to accumulate under stress and regulate translation in cells. Here we report the presence of tRNA-derived fragments in Entamoeba with tRNA halves being the most abundant. We further established that tRNA halves accumulate in the parasites upon different stress stimuli such as oxidative stress, heat shock, and serum starvation. We also observed differential expression of tRNA halves during developmental changes of trophozoite to cyst conversion with various tRNA halves accumulating during early encystation. In contrast to other systems, the stress response does not appear to be mediated by a few specific tRNA halves as multiple tRNAs appear to be processed during the various stresses. Furthermore, we identified some tRNAderived fragments are associated with Entamoeba Argonaute proteins, EhAgo2-2, and EhAgo23, which have a preference for different tRNA-derived fragment species. Finally, we show that tRNA halves are packaged inside extracellular vesicles secreted by amoeba. The ubiquitous presence of tRNA-derived fragments, their association with the Argonaute proteins, and the accumulation of tRNA halves during multiple different stresses including encystation suggest a nuanced level of gene expression regulation mediated by different tRNA-derived fragments in Entamoeba.
\end{abstract}

\title{
Importance
}

tRNA-derived fragments are small RNAs formed by the cleavage of tRNAs at specific positions. These have been reported in many organisms to modulate gene expression and thus regulate various cell functions. In the present study, we report for the first time the presence of tRNAderived fragments in Entamoeba. tRNA-derived fragments were identified by bioinformatics analyses of small RNA sequencing datasets from the parasites and also confirmed experimentally. We found that tRNA halves accumulated in parasites exposed to environmental stress or during developmental process of encystation. We also found that shorter tRNA-derived fragments are bound to Entamoeba Argonaute proteins, indicating that they may have a potential role in the Argonaute-mediated RNA-interference pathway which mediates robust gene silencing 
bioRxiv preprint doi: https://doi.org/10.1101/2021.09.30.462689; this version posted October 1, 2021. The copyright holder for this preprint

(which was not certified by peer review) is the author/funder, who has granted bioRxiv a license to display the preprint in perpetuity. It is made available under aCC-BY-ND 4.0 International license.

in Entamoeba. Our results suggest that tRNA-derived fragments in Entamoeba have a possible role in regulating gene expression during environmental stress. 


\section{$\underline{\text { Introduction }}$}

Entamoeba histolytica is a protozoan parasite with a biphasic lifecycle. A dormant cyst stage causes infection upon ingestion of contaminated food or water. The cysts transform into invasive trophozoites in the small intestine and proliferate upon reaching the colon (1). Entamoeba parasites can elicit disease symptoms if trophozoites invade the colonic wall, causing colitis. The life cycle is completed when trophozoites convert to cysts in the colon through a process known as encystation; these newly formed cysts can be excreted and spread to new hosts. Currently, the signals that cause the trophozoites to encyst in the colon are not completely understood (2). Our group has previously shown that extracellular vesicles (EVs) secreted by encysting parasites can promote encystation in Entamoeba parasites, though it was not ascertained what factors in the EV cargo were responsible for modulating encystation (3).

tRNA genes are extremely abundant in the amoeba genome with approximately 4,500 copies. The tRNA genes are organized in unique tandem repeats clustered into arrays that make up over $10 \%$ of the genome. Though the function of the arrays is not yet completely clear, there is evidence to show that these have a structural role in amoeba in the absence of classical telomeres $(4,5)$. Only four tRNA genes are outside of the arrays and the rest are exclusively found in the arrays. The existence of these low copy number tRNA genes does not affect efficient translation and no correlation has been found between codon usage and tRNA copy number.

tRNA-derived fragments (tRFs) are a class of small RNAs, approximately 16-40 bases in length, that have been identified in organisms from all domains of life (6). tRFs are produced through the cleavage of either precursor or mature tRNAs. Studies have shown that the cleavage can occur at any position on the tRNAs and is "context-dependent" (7). The best characterized tRFs are the tRNA halves, formed by the cleavage at the anticodon loop of mature tRNAs $(8,9)$. tRNA halves are 30 to 40 nucleotides in size and map to either the 5' or 3' end of the mature tRNA. In humans, angiogenin can cleave tRNAs at the anticodon loop during stress; the resultant tRNA halves have been shown to regulate translation (10). However, angiogenin independent mechanisms of tRNA halves have also been reported in human cells, as well as in other organisms that lack this protein $(11,12)$. Other tRFs are tRF-3s, tRF-5s, tRF-1s, and misc-tRFs 
(6). misc-tRFs include the internal-tRFs (itRFs) that span the internal regions of mature tRNAs, but do not map to either the 5' or 3' ends. tRFs generated from the 3'-end of mature tRNAs contain a 3'-CCA tail that is added to the precursor tRNA upon maturation.

One of the first studies in parasites describing tRNA fragments was performed in Giardia lamblia (13). Li et al. described a class of small RNAs, approximately 46nt in length, derived from the 3' end of mature tRNAs. These small RNAs, referred to as stress induced tRNAs (sitRNAs), contained a 3'-CCA tail and accumulated in the cell during encystation. Si-tRNAs are longer than tRNA halves and contain a cleavage site in the anticodon right arm. It was observed that si-tRNAs were generated indiscriminately from the entire tRNA family in the parasite. The authors further showed that si-tRNAs also accumulated during various stress-stimuli such as temperature shock and serum deprivation. More recently, tRNA halves have been shown to modulate translation during stress response in Trypanosoma brucei (12). Fricker et al. demonstrated that during serum starvation, $3^{\prime} \mathrm{tRNA}^{\mathrm{Thr}}$ accumulated in parasites and associated with ribosomes and polysomes to stimulate global protein translation (12). tRFs have also been reported in other parasites such as Trypanosoma cruzi and Plasmodium falciparum (14, 15). Moreover, Leishmania parasites and T. cruzi are known to package tRFs in their secreted exosomes suggesting that these parasites could be using tRFs as means of intercellular communication $(16,17)$.

tRFs have also been shown to associate with Argonaute (Ago) proteins in multiple organisms (18-21). This association suggests a possible role of tRFs in the RNA interference (RNAi) pathway. Indeed, there is increasing evidence demonstrating the loading of tRFs onto Argonaute proteins resulting in transcript cleavage based on sequence complementarity $(22,23)$. In humans, the 3 '-tRF from tRNA ${ }^{\text {GlyGCC }}$ has been shown to bind with Ago and lead to degradation of RPA1 to arrest B cell lymphoma (24). In the E. histolytica genome, three Ago proteins have been identified (EHI_125650,EHI_186850, and EHI_177170); EhAgo2-2 (EHI_125650) is the most highly expressed Ago protein in E. histolytica. We have previously demonstrated EhAgo2-2 binds to small RNA populations of $27 \mathrm{nt}$ or $31 \mathrm{nt}$ in size and mediates transcriptional gene silencing $(25,26)$. 
In our present work, we wished to identify if tRFs are present in Entamoeba and if there is a change in abundance in response to stress. We determined that tRFs can be identified in small RNA sequencing datasets of Entamoeba, and that these confirm experimentally by Northern blot experiments. Additionally, we determined that tRNA halves accumulate in amoeba during stress and encystation. Furthermore, we observed short tRFs (24-30nt in size) bound to Eh Argonaute proteins, suggesting a possible role in the Argonaute mediated gene silencing pathway. However, unlike in some other systems, such as Trypanosoma cruzi, where the stress response is mediated by elevation of only a few (or predominantly one) tRNA halves, stress conditions in amoeba leads to the elevation of a large subset of tRNA halves suggesting that the tRNA fragment pool in this parasite is more diverse and varied and possibly working in a redundant manner to carry out any molecular regulatory role after stress induction. 


\section{$\underline{\text { Results }}$}

\section{tRNA fragments identified in $E$. histolytica small RNA libraries and confirmed by Northern blot analyses}

To check for the presence of tRNA fragments in Entamoeba, we interrogated a previously published small RNA-seq datasets. These datasets consisted of small RNA libraries generated from E. histolytica parasites under basal growth conditions and during two different stresses (oxidative stress or heat stress $(27,28)$ ). For the purpose of our previous work, the libraries had been prepared by fractionating the total RNA into two size fractions - 15-30nt and 30-45nt. In the present study, the sequencing datasets from the two size-selected groups (for each sample) were combined together for subsequent analysis. For mapping the sequence reads, an index of $E$. histolytica tRNA sequences was first created from genomic sequences using tRNAscan-SE (29). Mature tRNAs contain a non-templated "CCA" tail at the 3' end. As such, tRNA fragments derived from the 3' ends of mature tRNAs contain a 3' CCA tail, whereas those derived from immature pre-tRNAs do not. A 'CCA' tail was added to the 3' terminal end of these predicted tRNA sequences to create a final tRNA index. The tRNA sequence index was used for mapping the sequencing datasets mentioned above, using Bowtie version 1.0.0 using a previously established pipeline $(27,30)$.

The total number of reads for each sample and the percentage of reads mapping to tRNAs is shown in Fig. 1A. Similar-sized libraries were obtained for the control and oxidative stress treated samples $(1,028,524$ and 1,446,363, respectively), and much higher for the heat-shock samples $(2,388,635)$. As published earlier, almost $40 \%$ of the reads were unique reads indicating that the sequencing was not saturated and likely missing rare species (27). Overall, around 3-6\% of the total sequence reads mapped to the tRNAs. The sequence reads that mapped to the tRNA sequences were selected and analyzed based on their lengths (Fig. 1B). A non-random distribution was observed, suggesting that the tRNA fragments were not generated by indiscriminate degradation. The peaks $\sim 35 \mathrm{nts}$ corresponded to the expected size for tRNA halves. Other tRFs, besides tRNA halves, were also present in the sequence reads as seen in peaks around 26 and 29nts. A majority of these tRNA fragments mapped to the 5' end of their respective tRNAs (> 90\% for each dataset). This trend is not uncommon, and for example it was 
recently reported that tRFs originating from the 5' end constituted around $86 \%$ of total tRFs in Plasmodium falciparum (14). However, there could have been a further bias generated during our library preparations which involved a 5' phosphate-dependent cloning step. As a result of this, tRFs containing 5 ' phosphate groups would be preferentially cloned compared to those formed by cleavage of tRNA resulting in a 5'-OH group.

The frequency of the tRNAs to which the reads mapped (note that these data show the cumulative mapping for all sequence reads of different sizes) is in Fig. 1C. The tRNA frequency is identical for the control sample compared to the samples under different stresses. The tRFs derived from 4 different tRNAs (tRNA ${ }^{\text {Ala_AGC }}, \mathrm{tRNA}^{\text {Ala_TGC }}, \mathrm{tRNA}^{\mathrm{Asp} \_\mathrm{GTC}}$, and tRNA ${ }^{\mathrm{Arg} \_\mathrm{TCT}}$ ) constituted around $80 \%$ of the total reads and tRNA ${ }^{\text {Ala_AGC }}$ was the most abundant tRNA to which the sequence reads mapped. Closer inspection of the sequence reads showed that the data are skewed by multiple copies of a few reads, similar to what has been reported for other systems.

Approximately $10 \%$ of the sequence reads mapped to the 3' end of tRNAs. We found that approximately $80 \%$ of these reads that mapped to the 3 ' end also contained a CCA tail, suggesting the tRFs were formed predominantly by the cleavage of mature tRNAs. The alignment of the sequence reads to representative tRNAs (tRNA ${ }^{\text {Ala_CGC }}$ and tRNA ${ }^{\text {Thr_TGT }}$,) in the integrated genome viewer (IGV) is in Fig. 1D (31). We are not comparing the tRFs from different samples but as a means to display the different tRNA-derived fragments in our dataset. Therefore, only the sequence reads from the oxidative-stress sample are used to show the mapping of different tRFs with respect to the parent tRNA. As seen in Fig 1D, the mapping is not randomly distributed but instead is localized to specific regions on the parent tRNA template (predominantly around the 5' end) which suggests that these reads are not degradation products. However, the diversity in the positions of termini suggests that some of the reads maybe the results of degradation/exonucleolytic process.

To confirm the presence of the tRFs in E. histolytica and to compare the tRF levels during different stress stimuli, northern blot analysis of total RNA was performed. Northern blot analysis for $5 \mathrm{tRNAF}^{\mathrm{Ala}{ }^{\mathrm{C} G C}}$ and $5 \mathrm{tRNAF}^{\mathrm{ARG} \_\mathrm{ACG}}$, displaying full length tRNA, and tRFs of different sizes are shown (Fig. 2A). tRNA halves (around 30-35nt in size) were the most abundant tRFs and were found to accumulate under the different stress conditions tested. Distinct bands corresponding to smaller tRFs were also observed but their relative amounts appeared to 
remain unaffected during the stress as compared to the control. We checked for tRNA halves corresponding to other tRNAs and found that in each case there was a stress-induced accumulation of tRNA halves (Fig. 2B). Oxidative stress following $\mathrm{H}_{2} \mathrm{O}_{2}$ treatment had the most significant impact on the accumulation of different tRNA halves. tRNA halves were not observed for 3' tRNA ${ }^{\text {Ala_CGC }}$. The presence of 5' tRNA ${ }^{\text {ALA }}$ portion but the absence of its corresponding 3' half has also been observed in other systems (32).

\section{tRNA halves accumulate in Entamoeba parasites during encystation}

Since Entamoeba encystation also involves stress on the parasites in the form of nutritional depletion, we wanted to determine if tRNA halves are regulated during this developmental process. Efficient encystation in E. histolytica has not been achieved in vitro and encystation of Entamoeba is studied using E. invadens $(33,34)$. We performed bioinformatics analyses of small RNA sequencing datasets (size selected RNA < 45nt in length) comparing developmental stages (trophozoites, early and late cysts, and excysting parasites) in E. invadens $(27,35)$. We observed that $\sim 1-4 \%$ of sequence reads mapped to the tRNA sequences (Fig. 3A). Analysis of the length distribution showed that tRFs were present in all the different developmental stages with peaks at around 25nt and 34nts (Fig. 3B). tRNA halves comprised a larger fraction of the total reads mapping to tRNAs in early cysts and excysting parasites compared to trophozoites. The $25 \mathrm{nt}$ sequence reads were the most abundant tRNA fragment species in trophozoites. As observed with E. histolytica parasites, a majority of the reads mapped to the 5 region of tRNAs (>80\%). The frequency of reads mapping to different tRNAs was found to be similar across different stages, with a few tRNAs (tRNA ${ }^{\text {Asp_GTC }}, \mathrm{tRNA}^{\mathrm{Gln} \_\mathrm{CTG}}, \mathrm{tRNA}^{\mathrm{Gln} \_\mathrm{TTG}}$, and tRNA ${ }^{\mathrm{Cys} \_\mathrm{GCA}}$ ) accounting for a majority of the sequence read-mapping (Fig. 3C). However, there was some apparent variability in the tRFs belonging to different stages of development. For example, reads mapping to tRNA ${ }^{\text {Asp_GTC }}$ constituted around $20 \%$ of total reads for trophozoites, early and late cysts but were only $5 \%$ of the reads in excysting parasites.

Northern blot analysis was carried out to compare the tRNA halves in E. invadens trophozoites and early cysts ( $24 \mathrm{hrs}$ following the induction of encystation). Similar to the stress response in E. histolytica, various tRNA halves accumulated during encystation. Representative northern blots for the 5' and 3' halves of tRNA ${ }^{\text {Thr_TGT }}$ and tRNA ${ }^{\text {Asn_GTT }}$ are shown (Fig. 3D). 
Northern blots carried out for 5' tRNA halves of tRNA ${ }^{\text {Arg_TCT }}, \mathrm{tRNA}^{\mathrm{Ala} \_\mathrm{CGC}}, \mathrm{tRNA}^{\mathrm{Gly} \text { GCC }}$, and tRNA ${ }^{\text {Ala_AGC }}$ are shown in Supplementary Fig S1.

\section{tRNA fragments in extracellular vesicles derived from $E$. histolytica}

In our previous work, we characterized EVs prepared from amoeba and analyzed their RNA cargo by sequencing (3). In the present study, the RNA-seq dataset from the size selected EV-RNA library was analyzed for tRFs as described above. Around $1 \%$ of the sequence reads mapped to tRNAs (Fig. 4A). Similar to what was observed in Fig. 1B, the length distribution of the cellular RNAs had peaks at around 26, 29, and 33nts Fig. 4B. The EV RNA had peaks at 29 and a more pronounced peak at 33nts, suggesting that tRNA halves are the most abundant tRNA derived fragments packaged in these EVs. Note that the protocol for EV preparation required the use of serum free media and therefore, both the EV and cellular RNA samples had been serum starved (3). As seen in Fig. 1D, serum starvation is one of the stressors that lead to accumulation of tRNA halves, similar to what was reported in different systems $(12,36)$. The frequency of the reads mapping to different tRNAs is in Fig. 4C. As previously seen, a majority of the reads mapped to a few tRNAs (tRNA ${ }^{\text {Ala_AGC }}, \mathrm{tRNA}^{\text {Ala_TGC }}, \mathrm{tRNA}^{\text {Arg_TCT }}, \mathrm{tRNA}^{\text {Asp_GTC }}$ ). We did not find any major differences in the IGV profiles for EV and cellular reads for the different tRNAs (data not shown). Northern blot analysis was performed to check for tRNA halves in $E$. histolytica cellular and EV RNA. EV preparation was done in serum free conditions to exclude contamination from exogenous exosomes in the serum (3). RNA isolated from the EVs was compared to cellular RNA isolated from parasites grown under similar serum free conditions. As seen in Fig. 4D, the 3 'tRNA ${ }^{\text {Asn_GTT }}$ tRNA half was observed in the EV RNA.

\section{tRNA-derived fragments are associated with $E$. histolytica Argonaute (EhAgo) proteins}

We previously demonstrated that two small RNA populations, 27 and 31nt in size, associate with EhAgo proteins $(25,26,28)$. Since Ago proteins have been reported to bind tRNA fragments in other systems $(23,37)$, we analyzed our sequencing data from size-fractionated total RNAs isolated from IPs of each of the 3 EhAgos: Ago2-1, Ago2-2 and Ago2-3. We found that the number of reads mapping to tRNA in IP-RNA was comparable to what we observed in 
whole cell sRNA samples. However, there were relatively higher mapping of reads from Ago2-3 (5.8\%) compared to those from Ago2-2 (3.8\%) or Ago 2-1 (1\%) (Fig. 5A). Length distribution of the mapped reads showed a non-random distribution with peaks at around 24 and $27 \mathrm{nt}$ for these tRNA fragments Fig. 5B. A majority of the sequence reads from the Ago2-3 samples were 24nt in length, whereas Ago2-2 and Ago2-1 were predominantly 27nt in size. Larger tRFs (e.g. tRNA halves around 33nt) were not observed binding to the Ago suggesting that Ago proteins might have a role in guiding shorter tRNA-derived fragments; this phenomena has been reported in other systems (37). The mapped reads were viewed on IGV against tRNA templates and two representative tRNAs- tRNA ${ }^{\text {Ala_TGC }}$ and tRNA ${ }^{\text {Arg_TCT }}$ are shown in Supplementary Fig. S2. The 5', tRNA-derived fragments were the most abundant group of tRFs binding to the three Ago proteins. Interestingly, Ago2-2 seems to bind to itRF species for different tRNAs. Though we did not observe itRFs in the cellular small RNA dataset (analyzed in Fig. 1), it is possible that the itRFs were enriched and stabilized in the Ago overexpression cell lines that were used for the IP experiments.

Northern blot analysis was carried out for the Ago-IP samples. We focused on association of tRFs with EhAgo2-2 \& EhAgo2-3 since those had the largest populations of mapping sequence reads. E. histolytica cell lines overexpressing Myc-tagged EhAgo2-2 or EhAgo2-3 were used for anti-Myc immunoprecipitation and the total associated RNA was isolated as described (28). Anti-Myc IP was carried out in parallel on untransfected E. histolytica trophozoites and RNA isolation performed to obtain a negative control. 5' tRNA ${ }^{\text {Thr_TGT }}$-derived fragments, around 27nt in length, were observed in RNA isolated from both EhAgo2-2 and EhAgo2-3 IPs, but with a stronger association with the latter (Fig. 5D). Compared to 5'tRNA $^{\text {Thr_TGT }}$-derived fragments, accumulation of other tRNA-derived fragments was seen at a much lower intensity not perceptible in our northern blots. We could not identify tRNA-derived fragments from 3'-tRNA ${ }^{\text {Thr_TGT }}$. This would make sense if binding of small RNAs to Ago proteins is dependent on the 5'phosphate groups on RNAs.

In mammalian cells, generation of tRNA halves have been reported by angiogenindependent and angiogenin-independent mechanisms (11). However, we could not find homologs of angiogenin in the amoebic genome based on sequence similarity by reciprocal blast searches or presence of a conserved domain by the use of Pfam (data not shown). This could suggest that 
an alternative tRNA cleavage mechanism is used to generate the tRNA halves in Entamoeba parasites. Mechanisms for tRNA generation is not uncommon and have been reported in various organisms. In yeasts, the RNase T2 family member, Rny1, can cleave tRNAs at the anticodon loop, to generate tRNA halves (38). In T. brucei, no known homologs of angiogenin or Rny1 exist (12). Entamoeba species have RNases that belong to the RNase T2 family and it is possible that these proteins could be involved in tRNA half biogenesis in response to stress. Further work would be required to investigate this.

A schematic of our findings of tRFs in amoeba is shown in Fig. 6. Cleavage of tRNAs gives rise to tRFs of distinct sizes that appear to be precisely generated and are not degradation products. When the parasites are treated to different stress inducers, tRNA halves are the most abundant tRFs that accumulate. Both 5' and 3' tRNA halves were generated in response to stress, and these corresponded to various tRNA isoacceptors. Moreover, tRNA halves are packaged inside extracellular vesicles where they could have a potential role in extracellular communication with other parasites. As reported in other systems, tRFs could bind to Ago proteins to initiate gene silencing in a manner similar to microRNAs. The role of the smaller tRFs in amebic biology is not clear although we found that some of the smaller tRFs associate with the EhAgo proteins. 


\section{Discussion}

tRNA genes are amongst the oldest genes that can be traced back to the putative RNA world that predated the separation of the three domains of life (39). It is no surprise then that tRNA-derived fragments have now been reported in Bacteria, Archaea, and Eukarya. Different classes of tRFs formed from the cleavage of either the mature or precursor tRNAs exist and have been shown to regulate gene expression in diverse contexts. Our present work provides evidence that multiple tRF species exist in amoeba and may have a potential to regulate gene regulation.

The amoeba genome is made up of arrays containing tandem repeats of tRNA clusters that make up over 105 of the genome (4). In spite of the high copy number of the tRNA genes, small RNA sequencing datasets in our labs have shown only around 5\% of reads mapping to the tRNA (35, 40). Around $80 \%$ of the tRFs originated from just four tRNAs- tRNA ${ }^{\text {Ala_AGC }}$, tRNA ${ }^{\text {Ala_TGC }}$, tRNA $^{\text {Asp_GTC }}$, and tRNA ${ }^{\text {Arg_TCT }}$. This has been reported in other systems as well $(41-43)$. However, the tRF abundance did not correlate with either the codon usage or the tRNA copy numbers. Again, this is not surprising and other groups have reported a similar absence of correlation between codon usage and tRF abundance $(44,45)$. Ghosh et.al. had carried out a comprehensive analysis of available E. histolytica genome datasets to determine codon bias and found that the genome is AT rich and overall A or T ending codons are strongly biased in the coding region. However, when only considering highly expressed genes, there was a clear bias for $\mathrm{C}$ ending codons, suggesting that these codons are translationally optimal for amoeba (46). Is there a correlation between the tRF fragment abundance (a majority originate from tRNAs with anticodons for G-ending codons) and translation efficiency in amoeba? The abundance of tRFs originating from different tRNAs varied during the development stages (analysis of E. invadens small RNA data Fig. 3C)- tRFs from tRNA ${ }^{\text {Gln_CTG }}$ were the most abundant tRFs in trophozoites whereas tRNA ${ }^{\text {Asp_GTC }}$ was the most abundant for late cysts. Further work would be required to understand if this tRF abundance is a cause or effect of the gene expression changes during stage conversion.

A majority of the tRFs identified through bioinformatic analysis mapped to the 5' end of tRNAs. Similar results have been reported elsewhere such as in Plasmodium falciparum (14). One reason 
for the overrepresentation of 5 'tRFs could be due to the nature of our library preparation- the libraries used in this analysis involved a 5' phosphate dependent cloning step (these were not prepared for tRF analysis). If the cleavage of the tRNA yields fragments with a 5'-OH group, these fragments would not be efficiently cloned in the library. For example, mature tRNAs cleaved in the middle, will yield two fragments: the fragment mapping to the 5 ' end will have the 5' phosphate group of the original tRNA. However, the other fragment (which would map to the 3' end) will have a 5'-OH group. Hence, the reads originating from the 5' end would be preferentially selected in our datasets. Northern blot analysis confirmed that both 5' and 3' tRNA halves were the most abundant tRFs observed during stress. For most tRNAs (e.g. tRNA ${ }^{\text {Thr_TGT }}$, tRNA $^{\text {Asn_GTT }}$, tRNA $^{\text {Gly_GCC }}$ etc) both 5' and 3' tRNA halves were confirmed by northern blotting. However, for tRNA ${ }^{\mathrm{Ala} \text { CGC }}$ which was one of the most abundant tRFs in the sequence analyses, only the 5' tRNA half was observed Fig. 2B. We found no tRNA where only the 3' tRNA half was observed in the northern blotting. Additional issues in our bioinformatic analysis could arise since we have included all reads that map to our selected tRNA sequences. A more thorough approach would omit sequence reads that also map elsewhere in the genome (47). However, in the present study, our aim was largely to check for the presence of tRNA fragments from existing sequencing data, rather than quantifying and comparing the tRNA fragments between samples.

Entamoeba are exposed to a variety of stresses during their life cycle inside the human host. The anaerobic parasite can survive extreme conditions such as high oxygen content during tissue invasion, reactive oxygen and nitrogen species released by the host immune response, and fluctuations in glucose availability $(48,49)$. The parasite employs a variety of strategies to cope with these stresses including the regulation of antioxidant protein expression (such as thioredoxin) (50), or $\mathrm{H}_{2} \mathrm{O}_{2}$ responsive proteins $(49,51)$. Recently tRFs have been discovered to be important regulators of gene expression, particularly during stress.

tRNA halves were the most abundant species of tRFs that accumulated during stress in amoeba. Cleavage of tRNAs at the anticodon loop to generate tRNA halves has been extensively reported in other organisms and parasites. In G. lamblia, 46nt-long si-tRNAs were reported to accumulate during encystation. Both si-tRNAs and slightly shorter tRNA halves ( $36 \mathrm{nt}$ in length) were 
observed in response to nutritional stress (13). Importantly, the si-tRNA were representatives of the entire tRNA family and not restricted to a few members. We observed a similar accumulation of tRNA halves in response to both encystation and various stress inducers (oxidative stress, serum starvation, or heat shock). As in Giardia, almost the entire tRNA family seems to accumulate in response to stress. However, unlike G. lamblia, the cleavage of tRNAs during stress or during encystation were identical in amoeba, yielding the same tRNA halves during both events. Furthermore, we observed both 5' and 3' halves in amoeba with a few tRNA halves $\left(\mathrm{tRNA}{ }^{\mathrm{Ala} \_\mathrm{AGC}}, \mathrm{tRNA}^{\mathrm{Ala} \_\mathrm{TGC}}, \mathrm{tRNA}{ }^{\mathrm{Asp} \_\mathrm{GTC}}\right.$, and tRNA ${ }^{\text {Arg_TCT }}$ accounting for around $90 \%$ of all sequence reads). This result was similar to what was observed in P. falciparum where approximately $90 \%$ of the total tRFs were derived from tRNAs coding for Pro, Phe, Asn, Gly, Cys, Gln, His, and Ala(14).

We had previously reported that extracellular vesicles secreted from encysting amoeba can modulate the encystation rates in recipient cells undergoing encystation (3). In our present work, we found that amoebic EVs contained tRNA halves and that tRNA half constituted $50 \%$ of reads mapping to tRNAs in EV small RNA dataset. Bioinformatics analyses of EVs and cellular RNAs showed no major difference in the tRNA half profiles between the two samples and it appears that the composition of tRNA halves in the cells is very similar to the cargo of secreted EVs. Further work would be required to assess if the tRNA halves in EVs are important for regulating encystation in the recipient cells. The RNA cargo of extracellular vesicles secreted by Trichomonas vaginalis was very well characterized and found to contain predominantly 5' tRNA halves. Moreover, the RNA content was seen to be internalized by the human host cells by lipidraft mediated endocytosis, suggesting that tRNA half carried by parasitic EVs could be a possible mode of extracellular gene-modulation (52). Similarly, in both old and new world Leishmania, tRFs are highly enriched in EVs and point to a conserved packaging of tRFs in EVs (53).

Amoebic parasites have been known to have a functional RNAi pathway mediated through a population of 27nt antisense RNAs $(25,40,54,55)$, including 3 Ago proteins which have been shown to bind sRNAs (26). Our data show that tRFs are also associated with the Ago proteins in amoeba. Further studies would be required to assess if the association of tRFs with the Ago proteins is an additional mode of gene silencing in amoeba. Kumar et. al. carried out a 
comprehensive analysis of Ago PAR-CLIP data and showed that 5' and 3'- tRFs can associate with target mRNAs in a mechanism reminiscent of microRNA gene silencing (37). With the bioinformatics analyses of our Ago-IP sequencing datasets, we also observed short reads (around 24-30nts in size) mapping to the tRNAs. The possibility of tRNA-derived fragments interacting with the RNAi pathways in amoeba offers an intriguing new role for the abundant tRNA genes in the amoebic genome.

Our data shows that various tRNA fragments are present in amoeba and that, similar to other organisms, accumulation of tRNA halves during stress-conditions is a mechanism that is conserved in amoeba. However, further work is required to figure out how exactly the tRNA halves accumulated during stress modulate protein synthesis and what the physiological role of this modulation is during amoebic infection. 


\section{Materials and methods}

\section{Parasite culture and cell lines.}

E. histolytica trophozoites (HM-1:IMSS) were grown axenically in TYI-S-33 (Trypticase, yeast extract, iron, and serum) medium in standard conditions described previously (56). E. invadens strain IP-1 was cultured in LYI-S-2 at $25^{\circ} \mathrm{C}$ (35). For encystation experiments, trophozoites at mid-log phase were iced, pooled, washed, and seeded into tubes in encystation medium (47\% LYI-LG), and incubated at $25{ }^{\circ} \mathrm{C}$. To measure encystation efficiency, total cells were counted using a hemocytometer before and after treatment with $0.1 \%$ sarkosyl. Parasites were transfected using the Attractene transfection reagent (Qiagen) to generate stable E. histolytica cell lines. All parasitetransfected lines were maintained at $6 \mu \mathrm{g} / \mathrm{ml} \mathrm{G} 418$.

\section{Extracellular vesicle isolation}

Parasites, grown to confluence in T25 flasks, were washed with serum-free TYI medium and incubated with $10 \mathrm{ml}$ serum-free TYI medium for $16 \mathrm{hrs}$ in an anaerobic chamber (BD GasPak EZ gas-generating container systems with GasPak EZ Campy container sachets; catalog number 260680). The conditioned medium was collected, centrifuged at 2,000 rpm, to remove cell debris and EVs pelleted using Total Exosome Isolation reagent (Invitrogen, Carlsbad CA, USA; catalog number 4478359) using manufacturer's protocol. The EV pellet was further purified using size-exclusion chromatography with SmartSEC TM Mini EV Isolation System, (Systems Biosciences, CA) (3).

\section{RNA isolation and analysis}

Cellular RNA was isolated using standard TRIzol (Invitrogen)-based protocol according to the manufacturer's protocol. For isolation of RNA from Immunoprecipitation samples, $300 \mu \mathrm{l}$ of TRIzol (Invitrogen) reagent was added to the final IP beads, and total RNA was isolated using manufacturer's protocol.

\section{Immunoprecipitation}

Immunoprecipitation experiments were done as previously described (28). Briefly, cell lysate was diluted with lysis buffer for a final protein concentration of $1 \mu \mathrm{g} / \mu \mathrm{l}$. 20-30 $\mu \mathrm{l}$ packed antiMyc beads (Thermo Scientific) were added to the IP mixture and rotated for 2 hours at $4{ }^{\circ} \mathrm{C}$. The beads were washed 6 times at $4{ }^{\circ} \mathrm{C}(5 \mathrm{~min}$ each) using a low stringency wash buffer (the basic 
lysis buffer plus $0.1 \%(\mathrm{v} / \mathrm{v})$ Tween-20, 0.1\% (v/v) NP-40, 1 mM PMSF and 0.5X HALT EDTA free protease inhibitors). After the final wash step, the beads were pelleted and used for RNA preparation using TRIzol reagent.

\section{Northern blot analysis}

Northern blot protocol was performed as previously described (25). $20 \mu \mathrm{g}$ RNA samples were separated on a denaturing $12 \%$ polyacrylamide gel and transferred to a membrane (Amersham ${ }^{\mathrm{TM}}$ Hybond $^{\mathrm{TM}}$-N+ Membrane, GE Healthcare). Probe DNA was 5'-end labeled by PNK reaction using $\gamma-\left[{ }^{32} \mathrm{P}\right]$-ATP and hybridized with the membrane in perfectHyb buffer (Sigma) overnight at $37^{\circ} \mathrm{C}$. The membrane was washed using low stringency condition $\left(2 \mathrm{X} \mathrm{SSC}, 0.1 \% \mathrm{SDS}\right.$ at $37^{\circ} \mathrm{C}$ for $20 \mathrm{~min}$ ) and medium stringency condition (1X SSC, $0.1 \% \mathrm{SDS}$ at $37^{\circ} \mathrm{C}$ for $20 \mathrm{~min}$ ).

Radioactive signal was detected using a Phosphor screen and imaged on a Personal Molecular Imager (Bio-Rad). The various probes used for corresponding tRFs are in the following table:

\begin{tabular}{r|ll}
\multicolumn{1}{rl}{ S.NO } & \multicolumn{1}{l}{ TRNA } & SEQUENCE \\
\hline $\mathbf{1}$ & tRNA $^{\text {ALA-CGC }}$ & AGCTCTACCAAATGAGCTACATCCCC \\
$\mathbf{2}$ & tRNA $^{\text {GLY-GCC }}$ & CAGGGATACGTCATACCATTGGACCACAAGTGC \\
$\mathbf{3}$ & tRNA $^{\text {GLY-GCC }}$ & GTGCACTTGCCGGGAATCGAACCCGGGTCTCATCC \\
$\mathbf{4}$ & tRNA $^{\text {ASN-GTT }}$ & CGCTTCCGCTTGGGATCGAACCAAGGACCTAACGG \\
$\mathbf{5}$ & tRNA $^{\text {ARG-TCT }}$ & TGCTCTTCCGACTGAGCTAACAAGGC \\
$\mathbf{6}$ & tRNA $^{\text {THR-TGT }}$ & ACAGATGCTCTACCGCTAAGCTAAACCGCC \\
$\mathbf{7}$ & tRNA $^{\text {THR-TGT }}$ & GGAGGCGACTATGGGGAACGAACCCATGACCTTCTGT \\
$\mathbf{8}$ & tRNA $^{\text {iMET-CAT }}$ & TGAGCCTGTCGCGCTACCACTGCGCCACCCCGCT \\
$\mathbf{9}$ & tRNA $^{\text {TRP-CCA }}$ & AGTCAGCTGCTCTGCCACTGAGCTAAGCCCCC \\
$\mathbf{1 0}$ & tRNA $^{\text {TRP-CCA }}$ & GGGTGAGGGCTAGAGGGATTGAACCACTGACCGGCTGA \\
$\mathbf{1 1}$ & tRNA $^{\text {GLY-GCC }}$ & CGCUUCCGCUUGGGAUCGAACCAAGGACCUAACGG \\
$\mathbf{1 2}$ & tRNA $^{\text {ALA-AGC }}$ & CGAATGCTCTACCATCTGAGCTACATCCCC \\
$\mathbf{1 3}$ & tRNA $^{\text {ALA-CGC }}$ & TGGAGATGAGGGGTATCGATCCCCTTGCCC \\
$\mathbf{1 4}$ & tRNA $^{\text {ARG-ACG }}$ & TGATGCGTTTCCATTGCGCCACAAGCCC \\
$\mathbf{1 5}$ & tRNA $^{\text {ARG-ACG }}$ & GGCGAGCTTGGCAGGGATCGAACCTGCAATCCCCAG \\
$\mathbf{1 6}$ & tRNA $^{\text {iMET-CAT }}$ & CAGCGGAGGGAAGTTTCGATCAACCGACCT \\
$\mathbf{1 7}$ & tRNA $^{\text {ASN-GTT }}$ & AGCCGTTCGCTCTGCCGACTGAGCCACGGAAGC \\
& &
\end{tabular}




\section{Bioinformatics analyses}

RNA sequencing data were analyzed using a previously described pipeline (40). Raw reads were processed using cutadapt (57). Sequences were then mapped to E. histolytica tRNA using Bowtie v1.2.2 (http://bowtie-bio.sourceforge.net) with the parameters - v1 and -best (30). tRNAscan-SE was used to predict tRNA genes from the genomes of E. histolytica and E. invadens (29). CCA was added to the genes at the 3' end.

tRNAscan-SE command used in this prediction run:

tRNAscan-SE -qQ --detail -o\# -m\# -f\# -1\# -c tRNAscan-SE.conf -b\# -s\# (fasta file)

\section{Acknowledgements}

The project was supported by funds to U.S. from the National Institutes of Health (R01AI121084 and R21-AI125764). We thank all members of the Singh lab for their help and contribution to this work. Its contents are solely the responsibility of the authors and do not necessarily represent the official views of the National Institutes of Health.

\section{References}

1. Stanley SL. 2001. Pathophysiology of amoebiasis. Trends Parasitol.

2. Eichinger D. 2001. Encystation in parasitic protozoa. Curr Opin Microbiol.

3. Sharma M, Morgado P, Zhang H, Ehrenkaufer G, Manna D, Singh U. 2020.

Characterization of extracellular vesicles from Entamoeba histolytica identifies roles in intercellular communication that regulates parasite growth and development . Infect Immun.

4. Clark CG, Ali IKM, Zaki M, Loftus BJ, Hall N. 2006. Unique organisation of tRNA genes in Entamoeba histolytica. Mol Biochem Parasitol 146:24-29.

5. Tawari B, Ali IKM, Scott C, Quail MA, Berriman M, Hall N, Clark CG. 2008. Patterns of 
evolution in the unique tRNA gene arrays of the genus Entamoeba. Mol Biol Evol.

6. Lee YS, Shibata Y, Malhotra A, Dutta A. 2009. A novel class of small RNAs: tRNAderived RNA fragments (tRFs). Genes Dev.

7. Telonis AG, Loher P, Magee R, Pliatsika V, Londin E, Kirino Y, Rigoutsos I. 2019. TRNA fragments show intertwining with mRNAs of specific repeat content and have links to disparities. Cancer Res.

8. Thompson DM, Lu C, Green PJ, Parker R. 2008. tRNA cleavage is a conserved response to oxidative stress in eukaryotes. RNA.

9. Levitz R, Chapman D, Amitsur M, Green R, Snyder L, Kaufmann G. 1990. The optional E. coli prr locus encodes a latent form of phage T4-induced anticodon nuclease. EMBO J.

10. Ivanov P, Emara MM, Villen J, Gygi SP, Anderson P. 2011. Angiogenin-Induced tRNA Fragments Inhibit Translation Initiation. Mol Cell.

11. Su Z, Kuscu C, Malik A, Shibata E, Dutta A. 2019. The role of ANGIOGENIN, or lack thereof, in the generation of stress-induced tRNA halves and of smaller tRNA fragments that enter Argonaute complexes. bioRxiv.

12. Fricker R, Brogli R, Luidalepp H, Wyss L, Fasnacht M, Joss O, Zywicki M, Helm M, Schneider A, Cristodero M, Polacek N. 2019. A tRNA half modulates translation as stress response in Trypanosoma brucei. Nat Commun.

13. Li Y, Luo J, Zhou H, Liao JY, Ma LM, Chen YQ, Qu LH. 2008. Stress-induced tRNAderived RNAs: A novel class of small RNAs in the primitive eukaryote Giardia lamblia. Nucleic Acids Res.

14. Wang Z, Wei C, Hao X, Deng W, Zhang L, Wang Z, Wang H. 2019. Genome-wide identification and characterization of transfer RNA-derived small RNAs in Plasmodium falciparum. Parasites and Vectors.

15. Garcia-Silva MR, Frugier M, Tosar JP, Correa-Dominguez A, Ronalte-Alves L, ParodiTalice A, Rovira C, Robello C, Goldenberg S, Cayota A. 2010. A population of tRNAderived small RNAs is actively produced in Trypanosoma cruzi and recruited to specific cytoplasmic granules. Mol Biochem Parasitol.

16. Lambertz U, Oviedo Ovando ME, Vasconcelos EJR, Unrau PJ, Myler PJ, Reiner NE. 2015. Small RNAs derived from tRNAs and rRNAs are highly enriched in exosomes from both old and new world Leishmania providing evidence for conserved exosomal RNA 
Packaging. BMC Genomics.

17. Bayer-Santos E, Lima FM, Ruiz JC, Almeida IC, Da Silveira JF. 2014. Characterization of the small RNA content of Trypanosoma cruzi extracellular vesicles. Mol Biochem Parasitol.

18. Hanada T, Weitzer S, Mair B, Bernreuther C, Wainger BJ, Ichida J, Hanada R, Orthofer M, Cronin SJ, Komnenovic V, Minis A, Sato F, Mimata H, Yoshimura A, Tamir I, Rainer J, Kofler R, Yaron A, Eggan KC, Woolf CJ, Glatzel M, Herbst R, Martinez J, Penninger JM. 2013. CLP1 links tRNA metabolism to progressive motor-neuron loss. Nature.

19. Kawamura Y, Saito K, Kin T, Ono Y, Asai K, Sunohara T, Okada TN, Siomi MC, Siomi H. 2008. Drosophila endogenous small RNAs bind to Argonaute 2 in somatic cells. Nature.

20. Nie Z, Zhou F, Li D, Lv Z, Chen J, Liu Y, Shu J, Sheng Q, Yu W, Zhang W, Jiang C, Yao Y, Yao J, Jin Y, Zhang Y. 2013. RIP-seq of BmAgo2-associated small RNAs reveal various types of small non-coding RNAs in the silkworm, Bombyx mori. BMC Genomics.

21. Cole C, Sobala A, Lu C, Thatcher SR, Bowman A, Brown JWS, Green PJ, Barton GJ, Hutvagner G. 2009. Filtering of deep sequencing data reveals the existence of abundant Dicer-dependent small RNAs derived from tRNAs. RNA.

22. Haussecker D, Huang Y, Lau A, Parameswaran P, Fire AZ, Kay MA. 2010. Human tRNA-derived small RNAs in the global regulation of RNA silencing. RNA.

23. Kuscu C, Kumar P, Kiran M, Su Z, Malik A, Dutta A. 2018. tRNA fragments (tRFs) guide Ago to regulate gene expression post-transcriptionally in a Dicer-independent manner. RNA.

24. Maute RL, Schneider C, Sumazin P, Holmes A, Califano A, Basso K, Dalla-Favera R. 2013. TRNA-derived microRNA modulates proliferation and the DNA damage response and is down-regulated in B cell lymphoma. Proc Natl Acad Sci U S A.

25. Zhang H, Ehrenkaufer GM, Pompey JM, Hackney JA, Singh U. 2008. Small RNAs with 5 '-polyphosphate termini associate with a piwi-related protein and regulate gene expression in the single-celled eukaryote Entamoeba histolytica. PLoS Pathog.

26. Zhang H, Tran V, Manna D, Ehrenkaufer G, Singh U. 2019. Functional Characterization of Entamoeba histolytica Argonaute Proteins Reveals a Repetitive DR-Rich Motif Region That Controls Nuclear Localization . mSphere. 
27. Zhang H, Ehrenkaufer GM, Manna D, Hall N, Singh U. 2015. High throughput sequencing of Entamoeba 27nt small RNA population reveals role in permanent gene silencing but no effect on regulating gene expression changes during stage conversion, oxidative, or heat shock stress. PLoS One.

28. Zhang H, Ehrenkaufer GM, Hall N, Singh U. 2020. Identification of oligo-adenylated small RNAs in the parasite Entamoeba and a potential role for small RNA control. BMC Genomics.

29. Lowe TM, Eddy SR. 1996. TRNAscan-SE: A program for improved detection of transfer RNA genes in genomic sequence. Nucleic Acids Res.

30. Langmead B, Trapnell C, Pop M, Salzberg SL. 2009. Ultrafast and memory-efficient alignment of short DNA sequences to the human genome. Genome Biol.

31. Robinson JT, Thorvaldsdóttir H, Winckler W, Guttman M, Lander ES, Getz G, Mesirov JP. 2011. Integrative genomics viewer. Nat Biotechnol.

32. Cognat V, Morelle G, Megel C, Lalande S, Molinier J, Vincent T, Small I, Duchene AM, Marechal-Drouard L. 2017. The nuclear and organellar tRNA-derived RNA fragment population in Arabidopsis thaliana is highly dynamic. Nucleic Acids Res.

33. Mitra BN, Pradel G, Frevert U, Eichinger D. 2010. Compounds of the upper gastrointestinal tract induce rapid and efficient excystation of Entamoeba invadens. Int $\mathbf{J}$ Parasitol.

34. Vazquezdelara-Cisneros LG, Arroyo-Begovich A. 1984. Induction of Encystation of Entamoeba invadens by Removal of Glucose from the Culture Medium. J Parasitol.

35. Ehrenkaufer GM, Weedall GD, Williams D, Lorenzi HA, Caler E, Hall N, Singh U. 2013. The genome and transcriptome of the enteric parasite Entamoeba invadens, a model for encystation. Genome Biol.

36. Yamasaki S, Ivanov P, Hu GF, Anderson P. 2009. Angiogenin cleaves tRNA and promotes stress-induced translational repression. J Cell Biol.

37. Kumar P, Anaya J, Mudunuri SB, Dutta A. 2014. Meta-analysis of tRNA derived RNA fragments reveals that they are evolutionarily conserved and associate with AGO proteins to recognize specific RNA targets. BMC Med.

38. Thompson DM, Parker R. 2009. The RNase Rny1p cleaves tRNAs and promotes cell death during oxidative stress in Saccharomyces cerevisiae. J Cell Biol. 
39. Alberts B, Johnson A, Lewis J, Raff M, Roberts K, Walter P. 2002. The RNA World and the Origins of Life.

40. Zhang H, Ehrenkaufer GM, Hall N, Singh U. 2013. Small RNA pyrosequencing in the protozoan parasite Entamoeba histolytica reveals strain-specific small RNAs that target virulence genes. BMC Genomics.

41. Chen Q, Yan M, Cao Z, Li X, Zhang Y, Shi J, Feng GH, Peng H, Zhang X, Zhang Y, Qian J, Duan E, Zhai Q, Zhou Q. 2016. Sperm tsRNAs contribute to intergenerational inheritance of an acquired metabolic disorder. Science (80- ) 351:397-400.

42. Krishna S, Yim DG, Lakshmanan V, Tirumalai V, Koh JL, Park JE, Cheong JK, Low JL, Lim MJ, Sze SK, Shivaprasad P, Gulyani A, Raghavan S, Palakodeti D, DasGupta R. 2019. Dynamic expression of tRNA-derived small RNAs define cellular states. EMBO Rep 20:e47789.

43. Lakshmanan V, Sujith TN, Bansal D, Shivaprasad P V., Palakodeti D, Krishna S. 2021. Comprehensive annotation and characterization of planarian tRNA and tRNA-derived fragments (tRFs). RNA 27:477-495.

44. Gebetsberger J, Zywicki M, Künzi A, Polacek N. 2012. TRNA-derived fragments target the ribosome and function as regulatory non-coding RNA in Haloferax volcanii. Archaea 2012.

45. Guan L, Karaiskos S, Grigoriev A. 2020. Inferring targeting modes of Argonaute-loaded tRNA fragments. RNA Biol 17:1070-1080.

46. Ghosh TC, Gupta SK, Majumdar S. 2000. Studies on codon usage in Entamoeba histolytica. Int J Parasitol 30:715-722.

47. Magee R, Rigoutsos I. 2020. On the expanding roles of tRNA fragments in modulating cell behavior. Nucleic Acids Res.

48. Olivos-García A, Saavedra E, Nequiz M, Santos F, Luis-García ER, Gudiño M, PérezTamayo R. 2016. The oxygen reduction pathway and heat shock stress response are both required for Entamoeba histolytica pathogenicity. Curr Genet.

49. Nagaraja S, Ankri S. 2018. Utilization of different omic approaches to unravel stress response mechanisms in the parasite Entamoeba histolytica. Front Cell Infect Microbiol.

50. Jeelani G, Nozaki T. 2016. Entamoeba thiol-based redox metabolism: A potential target for drug development. Mol Biochem Parasitol. 
51. Pearson RJ, Morf L, Singh U. 2013. Regulation of H2O2 Stress-responsive genes through a novel transcription factor in the protozoan pathogen Entamoeba histolytica. J Biol Chem.

52. Artuyants A, Campos TL, Rai AK, Johnson PJ, Dauros-Singorenko P, Phillips A, SimoesBarbosa A. 2020. Extracellular vesicles produced by the protozoan parasite Trichomonas vaginalis contain a preferential cargo of tRNA-derived small RNAs. Int J Parasitol 50:1145-1155.

53. Lambertz U, Oviedo Ovando ME, Vasconcelos EJR, Unrau PJ, Myler PJ, Reiner NE. 2015. Small RNAs derived from tRNAs and rRNAs are highly enriched in exosomes from both old and new world Leishmania providing evidence for conserved exosomal RNA Packaging. BMC Genomics 16:1-26.

54. Zhang H, Alramini H, Tran V, Singh U. 2011. Nucleus-localized antisense small RNAs with 5'-polyphosphate termini regulate long term transcriptional gene silencing in Entamoeba histolytica G3 strain. J Biol Chem.

55. Vega VL, Rodríguez-Silva M, Frey T, Gehrmann M, Diaz JC, Steinem C, Multhoff G, Arispe N, De Maio A. 2008. Hsp70 Translocates into the Plasma Membrane after Stress and Is Released into the Extracellular Environment in a Membrane-Associated Form that Activates Macrophages. J Immunol.

56. Diamond LS, Harlow DR, Cunnick CC. 1978. A new medium for the axenic cultivation of entamoeba histolytica and other entamoeba. Trans R Soc Trop Med Hyg.

57. Martin M. 2011. Cutadapt removes adapter sequences from high-throughput sequencing reads. EMBnet.journal. 


\section{Figure Legends:}

\section{Figure 1: Bioinformatics analyses of small RNA sequencing dataset from $E$. histolytica} demonstrates the presence of tRFs. (A) Small RNA sequences generated from size selected libraries from $E$. histolytica in either basal or stress conditions were mapped to tRNA sequences using Bowtie. Chart showing the percentage of sequence reads that map to tRNA in different samples. (B) Length distribution of the different reads that mapped to tRNA sequences. (C) Frequency of reads mapping to the four most abundant tRNAs seen. (D) Representatitive IGV mapping of sequence reads from E. histolytica (oxidative stress sample) mapping against tRNA templates- $t R N A^{\text {Ala_CGC }}$ and tRNA ${ }^{\text {Thr_TGT }}$. The top of each IGV mapping shows the size of the parent tRNA template (72 or $73 \mathrm{nt}$ for $\mathrm{tRNA}^{\text {Ala_CGC }}$ and $\mathrm{tRNA}{ }^{\text {Thr_TGT }}$ respectively). The coverage bar in grey displays the depth of the reads at each locus as a bar chart. Below this, the sequence alignment tracks represent each sequence read aligned to the parent tRNA, whose sequence is shown as the "Reference Sequence" below. As seen for a majority of tRNAs, both 5' and 3' tRNA halves were observed for tRNA ${ }^{\text {Thr }}{ }^{\text {TGT }}$. However, only the 5' tRNA half was observed for tRNA ${ }^{\text {Ala_CGC }}$.

\section{Figure 2: Northern blot analysis of tRNA fragments from $E$. histolytica shows accumulation} of tRNA halves during stress. (A) Total RNA was prepared from E. histolytica parasites under basal condition or stressed with oxidative stress $\left(\mathrm{H}_{2} \mathrm{O}_{2}\right)$, Heat shock (42 C) or serum starvation, and analyzed by northern blotting. Representative blots of 5 tRNAF $^{\text {Ala_CGC }}$ and 5 tRNAF ${ }^{\text {ARG_ACG }}$ show the presence of abundant tRNA halves during stress as well as smaller tRFs of distinct sizes. (B) Northern blots showing tRNA halves for different tRNAs. Both 5' and 3' tRNA halves were seen to accumulate in response to the different stresses, for tRNA ${ }^{\text {Thr_TGT }}$, RRNAF $^{\text {Gly_GCC }}$, tRNAF ${ }^{\text {Asn_GTT }}$ whereas only the 5' tRNA half was observed for tRNAF ${ }^{\text {Ala_CGC }}$.

Figure 3: tRNA halves accumulate during encystation of $\boldsymbol{E}$. invadens. (A) Small RNA sequences generated from size selected libraries from $E$. invadens parasites in different stagesTrophozoites, $24 \mathrm{~h}$ cysts, $72 \mathrm{~h}$ cysts or excysting parasites at $8 \mathrm{~h}$. Chart shows percentage of reads mapping to tRNA. (B) Length distribution of reads mapping to tRNA sequences. (C) Frequency of reads mapping to most abundant tRNAs. (D) Northern blot analysis of total RNA from $24 \mathrm{~h}$ cysts and trophozoites probed for tRNA halves. Both 5' and 3' halves were seen to be 
accumulated in the early cysts stage compared to the trophozoites

Figure 4: tRNA fragments are identified in small RNA sequence datasets obtained from size selected libraries of EVs secreted by E. histolytica. (A) Percentage of reads mapping to tRNA. (B) Length distribution (C) RNA frequency. (D) Northern blot analysis of EV and cellular RNA prepared from E. histolytica parasites. 3 'tRNA ${ }^{\text {Asn_GTT }}$ was seen to be packaged in EV.

Figure 5: tRFs associate with the three $E$ Aggo proteins in amoeba. (A) IP from Myc-tagged overexpression cell lines for the three EhAgo proteins were performed and RNA from beads isolated. Small RNA libraries were prepared by size selection and sequenced. Chart showing the percentage of sequence reads that map to tRNA in different samples. (B) Length distribution of the different reads that mapped to tRNA sequences. (C) Frequency of reads mapping to the most abundant tRNAs. (D) Norther blot analysis of IP-RNA prepared from myc-EhAgo2-2 and mycEh-Ago2-3 overexpression cells lines. IP-HM1 refers to IP performed with un-transfected HM1 control cells. Cellular RNA was used as a positive control. tRFs were observed in both IP-Ago22 and IP-Ago2-3 samples for $5^{`}$ tRNA $^{\text {Thr_TGT }}$ but not for $3^{`}$ tRNA $^{\text {Thr_TGT }}$.

Figure 6: Schematic outlining potential roles of tRFs in amoeba. Both 5' and 3' tRFs are produced in amoeba. tRNA halves get accumulated during stress and during encystation and these can be subsequently packed into EVs for extracellular communication. Smaller tRFs - 3', 5' or itRFs are also present in Amoeba and are seen to bind to Ago proteins for possible roles in gene silencing.

\section{Supplementary Figures}

Fig. S1: Northern blot analysis of total RNA from $24 \mathrm{~h}$ cysts and trophozoites probed for tRNA halves. tRNA halves were seen to accumulate for the different tRNAs probed.

Fig. S2: IGV mapping of reads mapping against tRNA templates- $\mathrm{tRNA}^{\text {Ala_TGC }}$ and tRNA ${ }^{\text {Arg_TCT }}$. A $24 n t 5$ ' tRF is seen to be associated with all Ago proteins for tRNA ${ }^{\text {Ala_TGC }}$. An itRF species is seen to be associated with Ago2-2 for both tRNAs. 


\section{Fig 1}

A

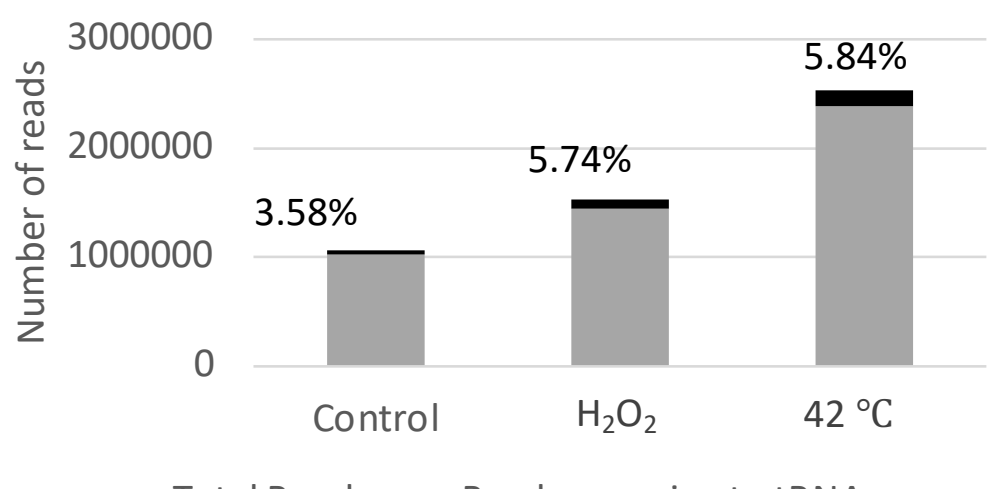

Total Reads Reads mapping to tRNA
B

All reads mapping to tRNA

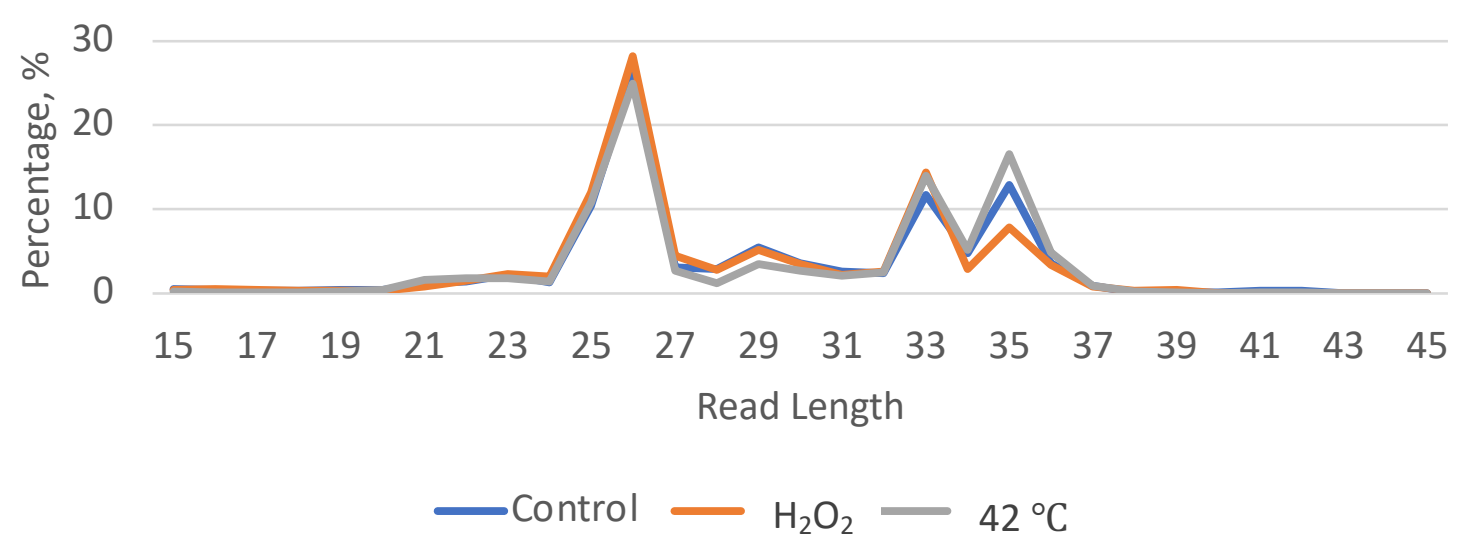

C

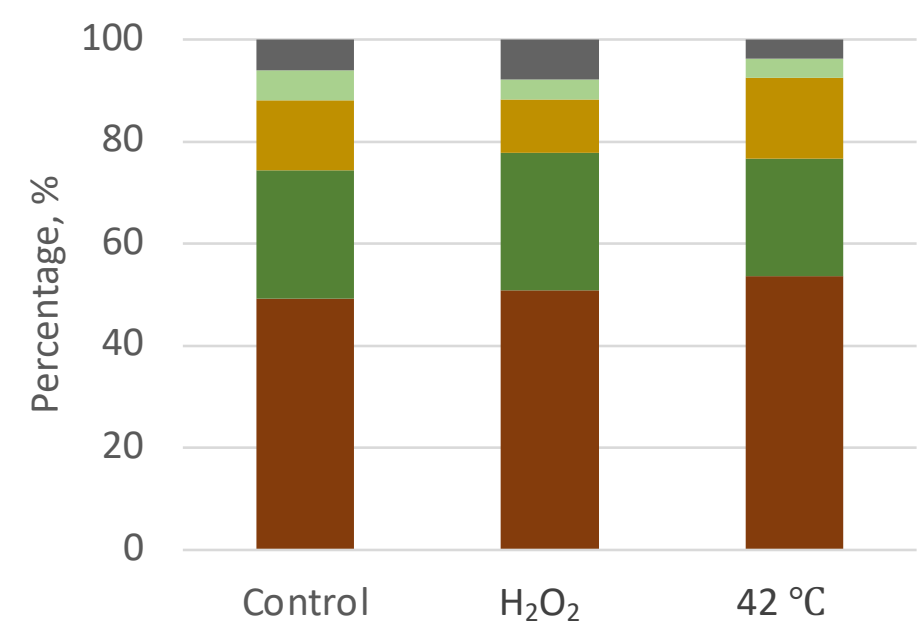

AlaAGC $\square$ AlaTGC $\square$ AspgTC ArgTCT $\square$ Others 
Fig 1 D

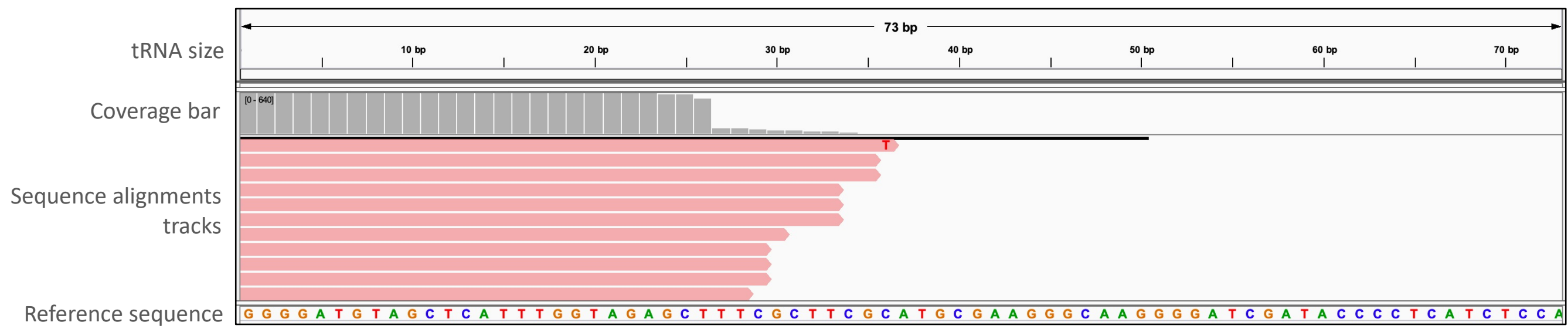

tRNAAla_CGC

Reference sequence

GGGGATGTAGCTCATTTGGTAGAGCTTTCGCTTCGCATGCGAAGGGCAAGGGGATCGATACCCGTCATCTCCA

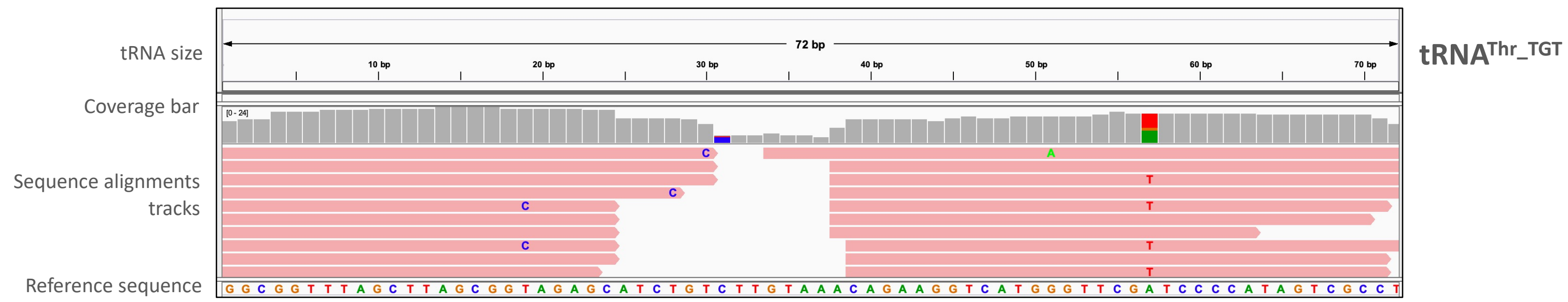




\section{Fig 2A}
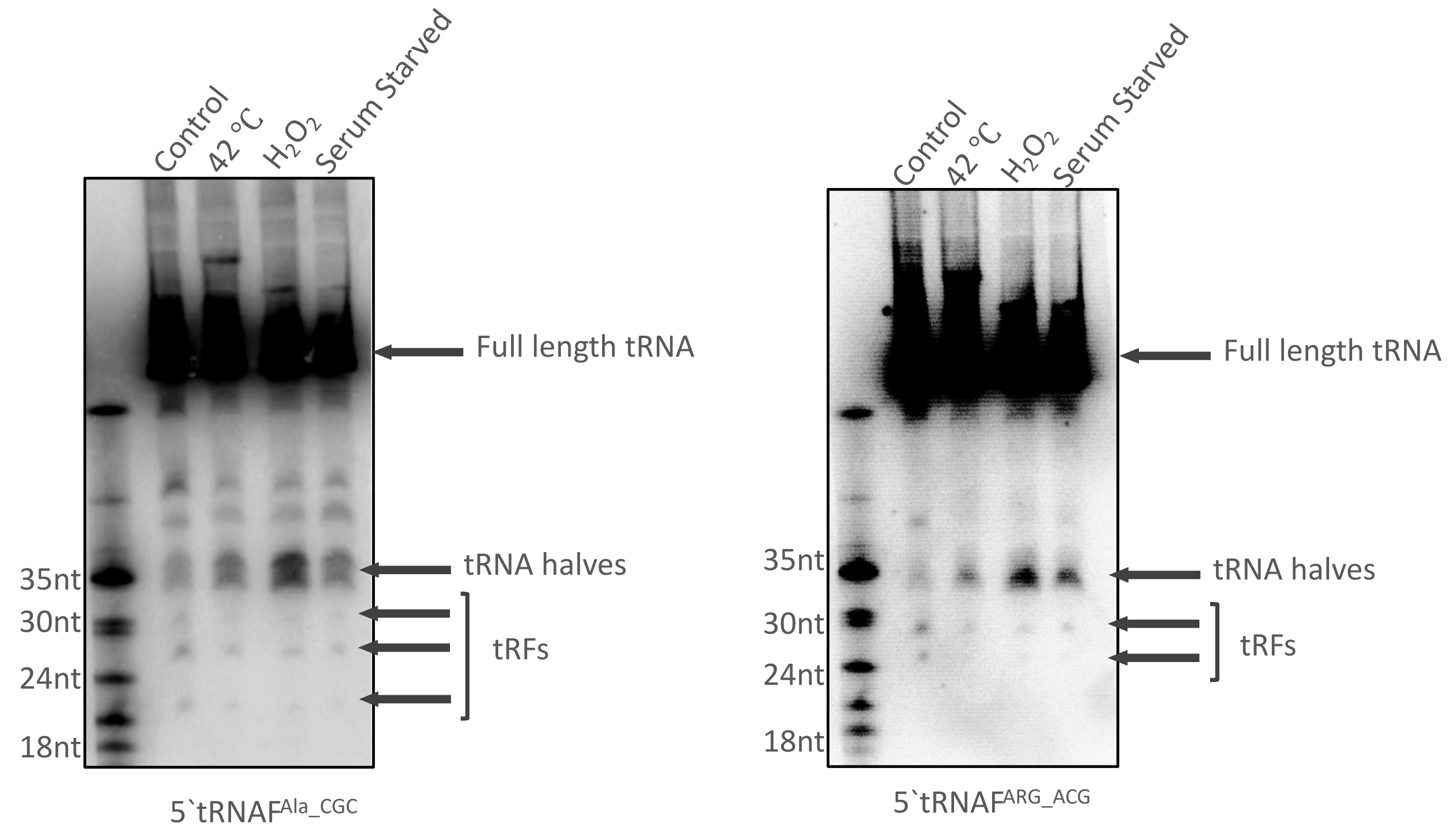
Fig 2B
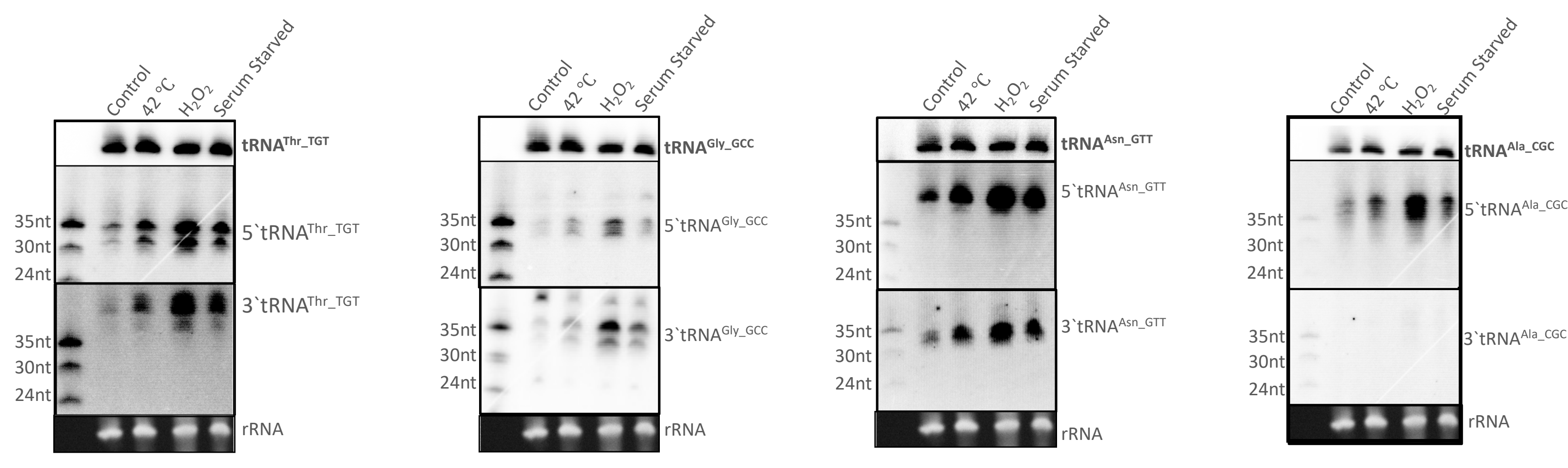
Fig 3

A

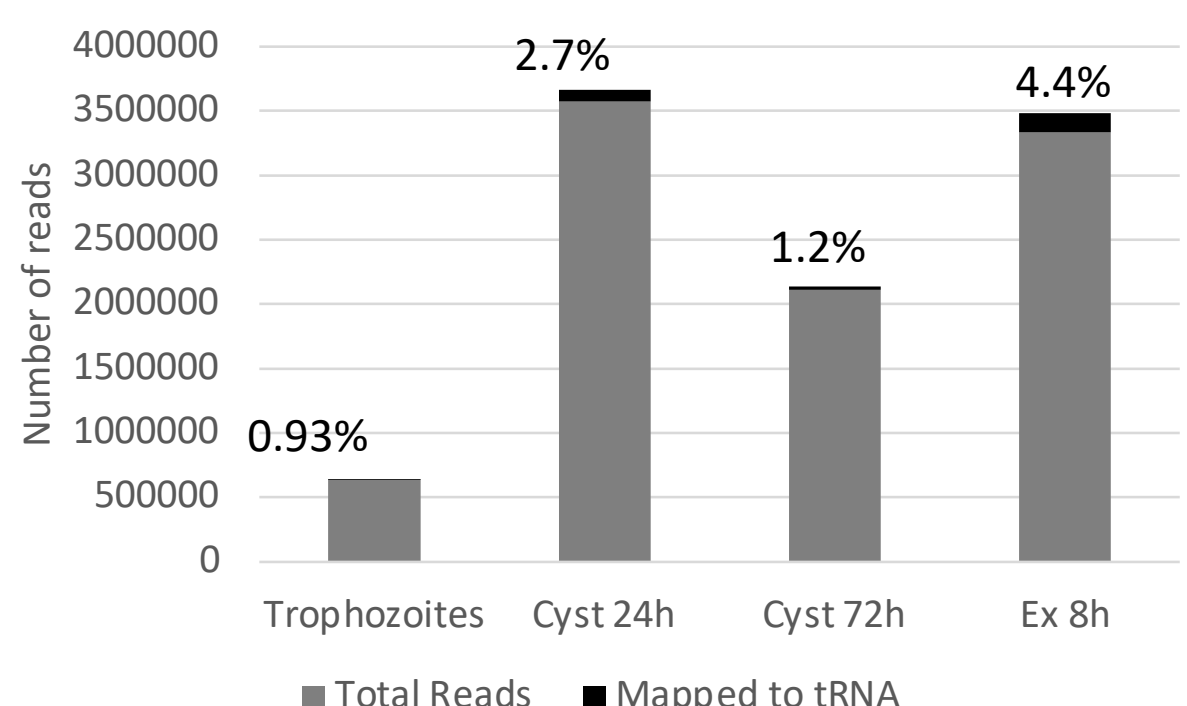

C

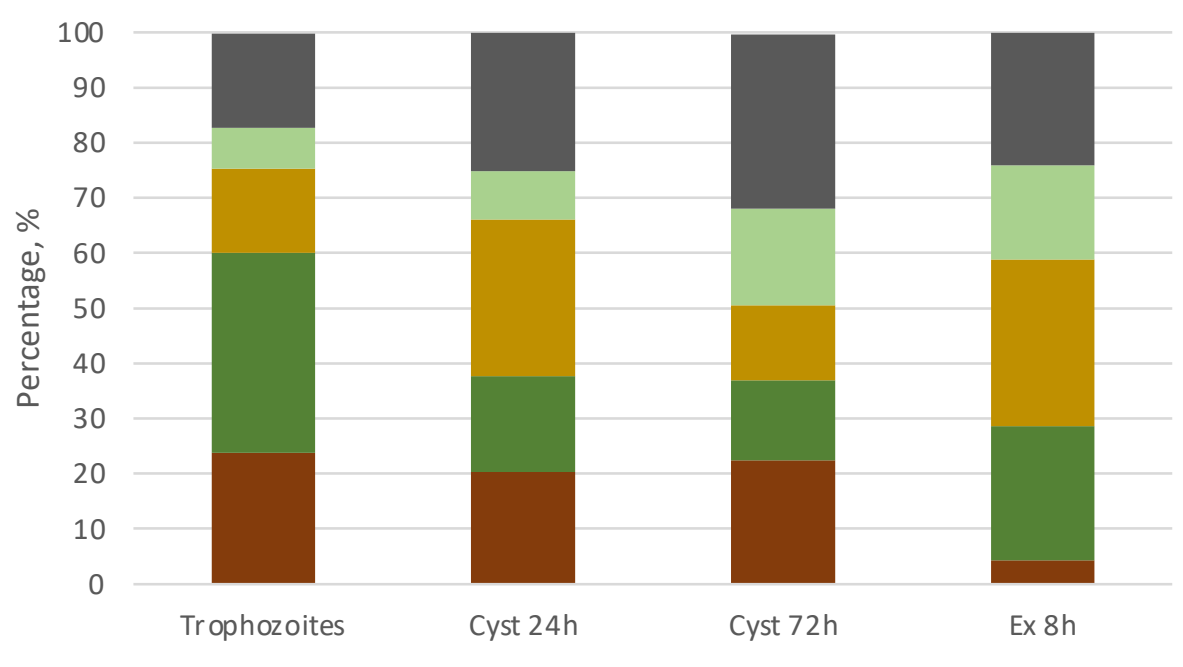

B

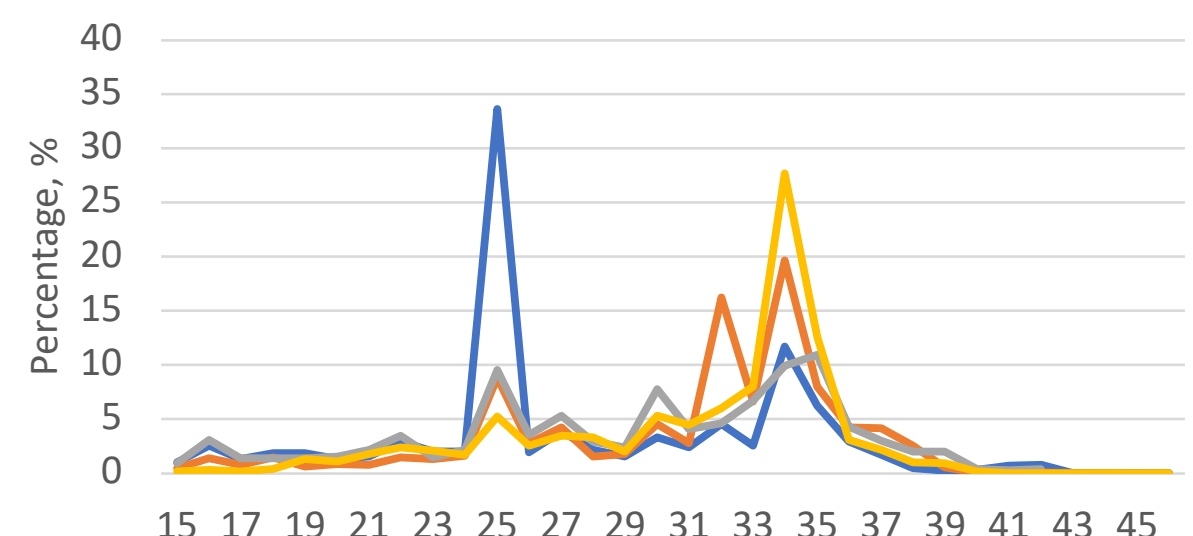

$\begin{array}{llllllllllllllll}15 & 17 & 19 & 21 & 23 & 25 & 27 & 29 & 31 & 33 & 35 & 37 & 39 & 41 & 43 & 45\end{array}$ Read Length

-Trophozoites Cyst 24h—Cyst 72h Ex 8h

\section{D}

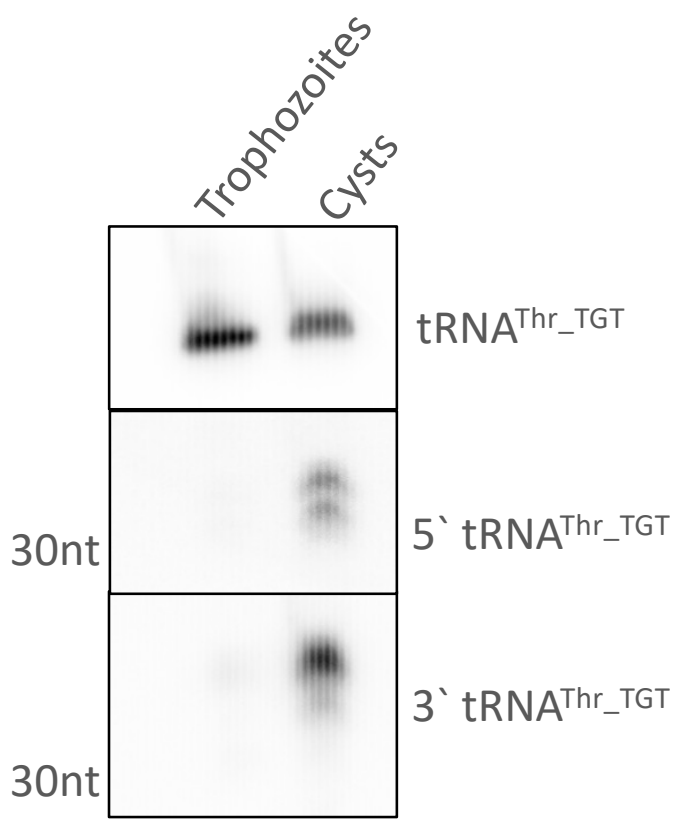

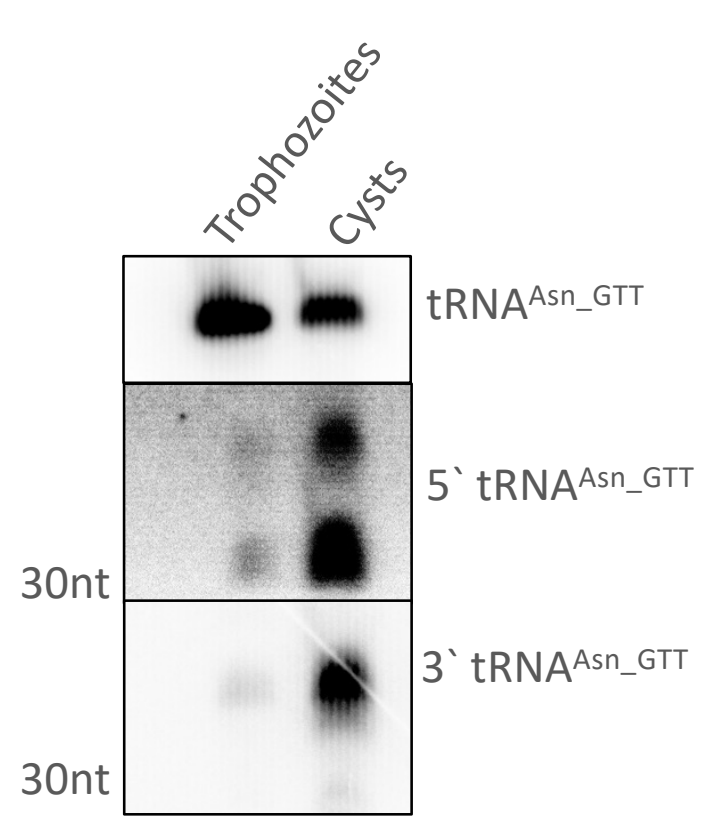




\section{Fig 4}

A

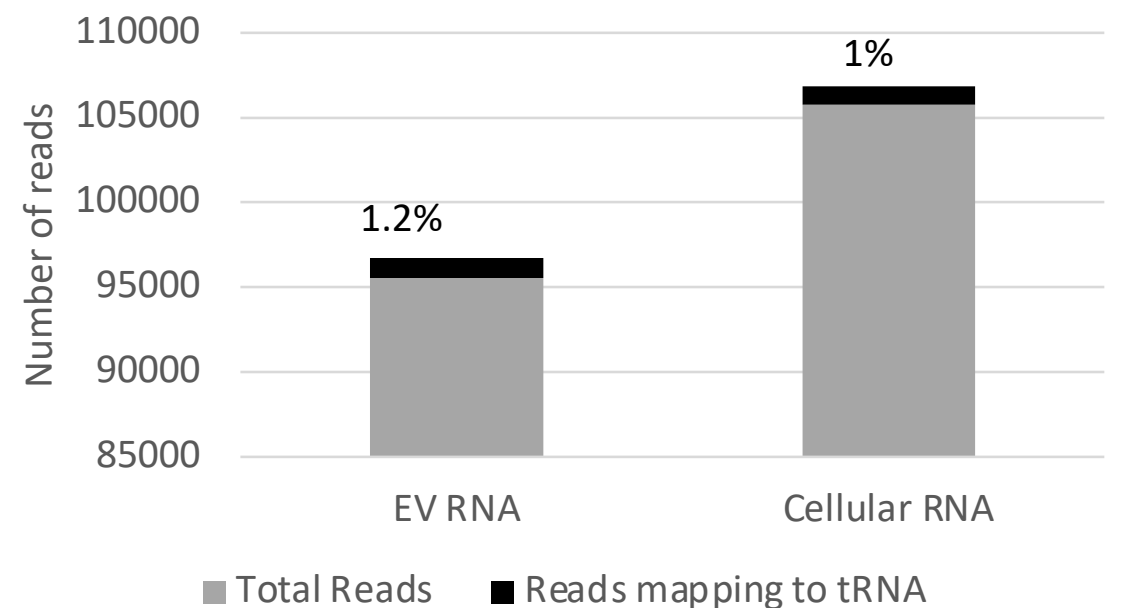

C

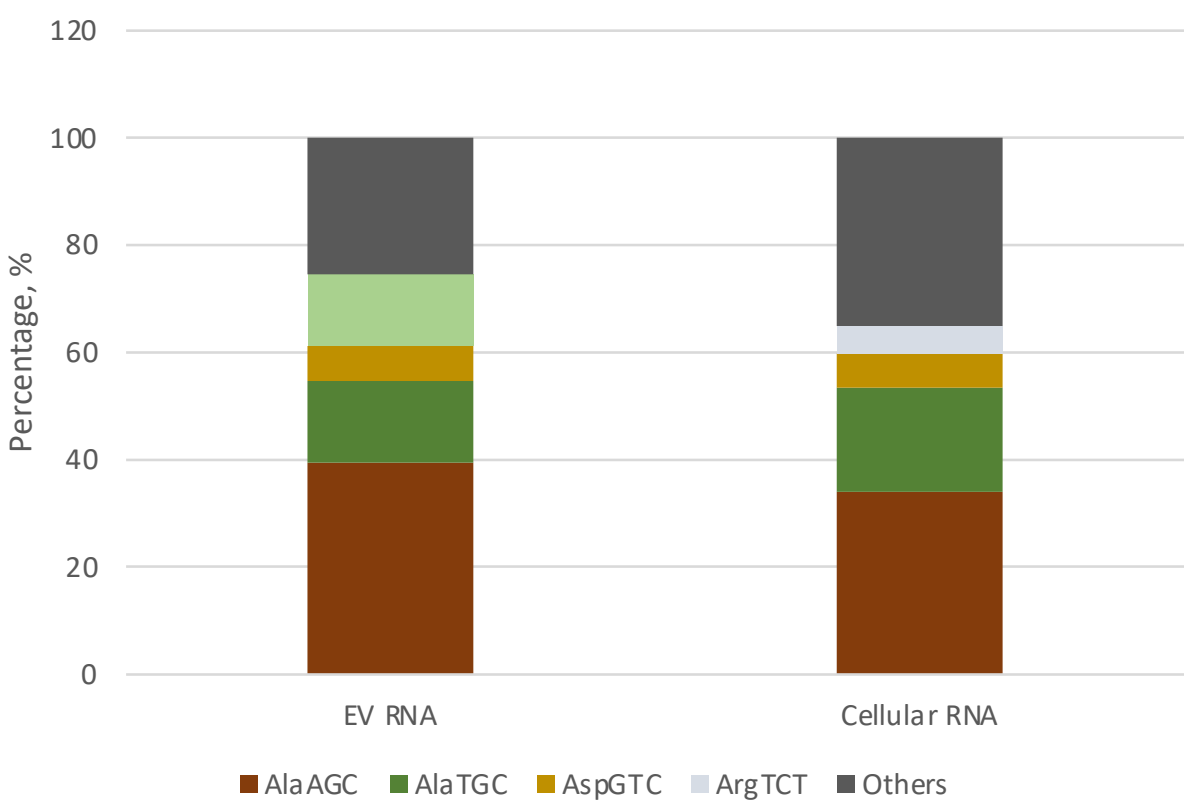

B

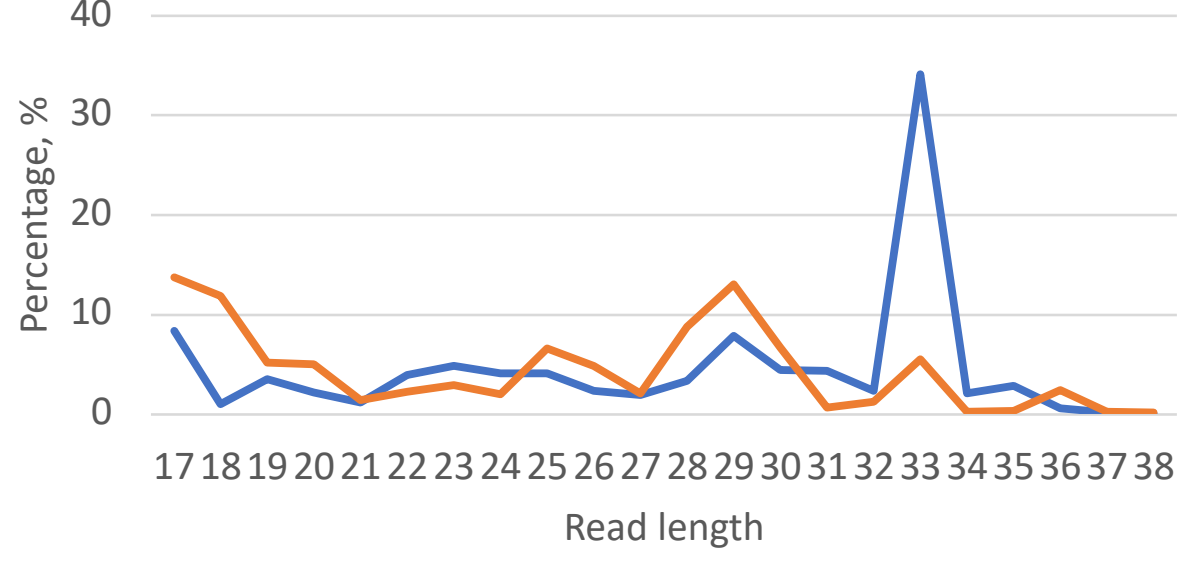

EV RNA Cellular RNA

D

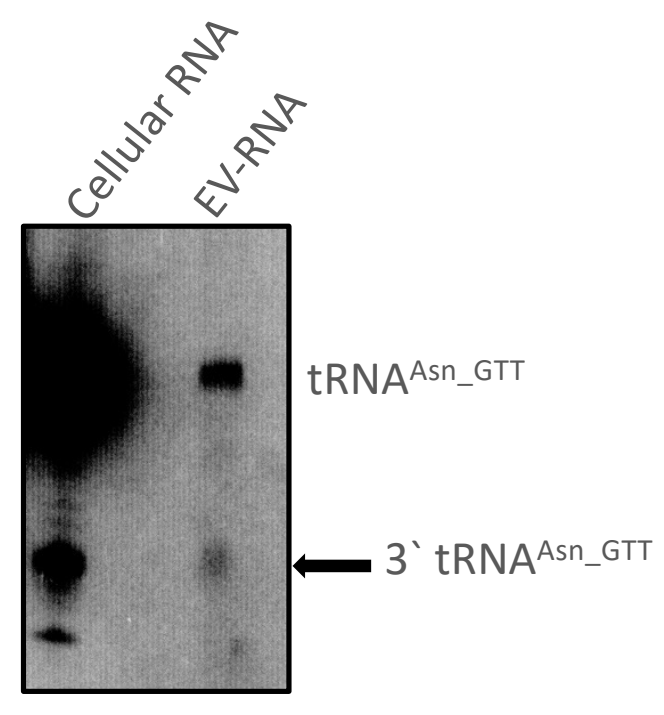




\section{Fig 5}

A

$5.82 \%$

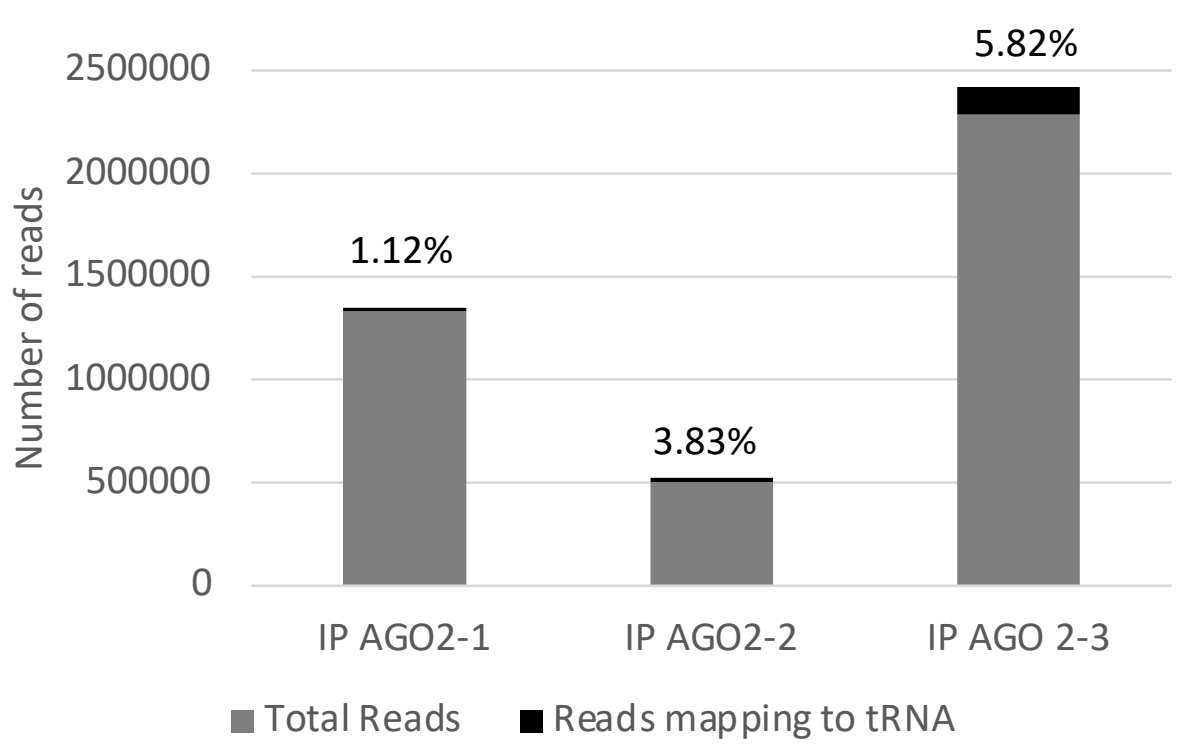

C

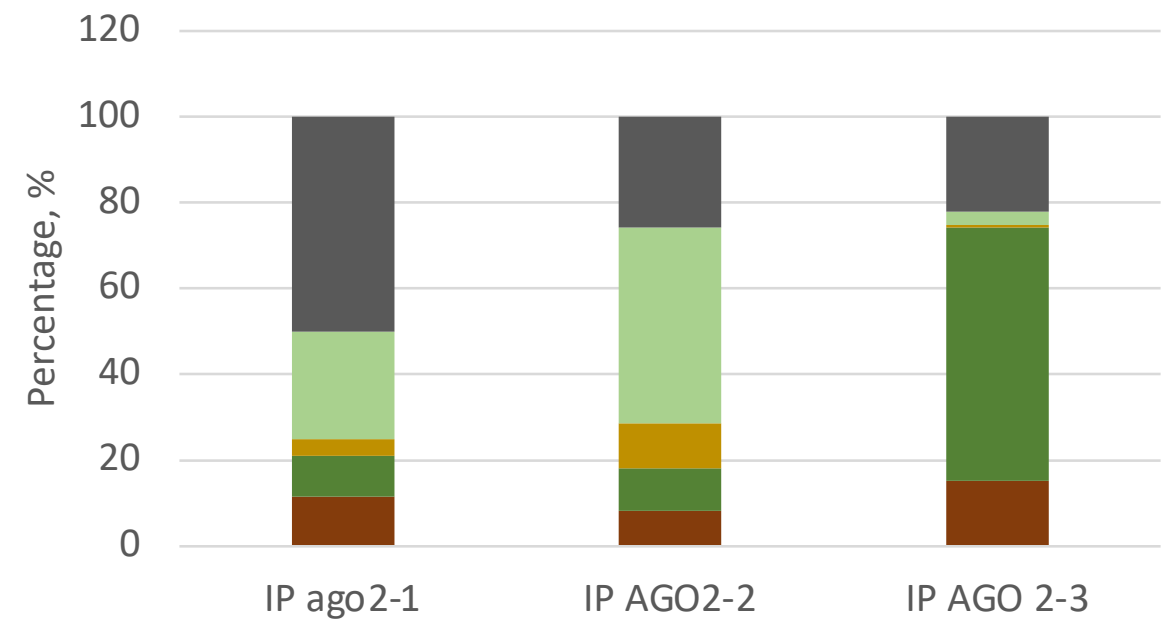

- AlaAGC

AlaTGC

- LeUTAA TyrGTA

- Others
B

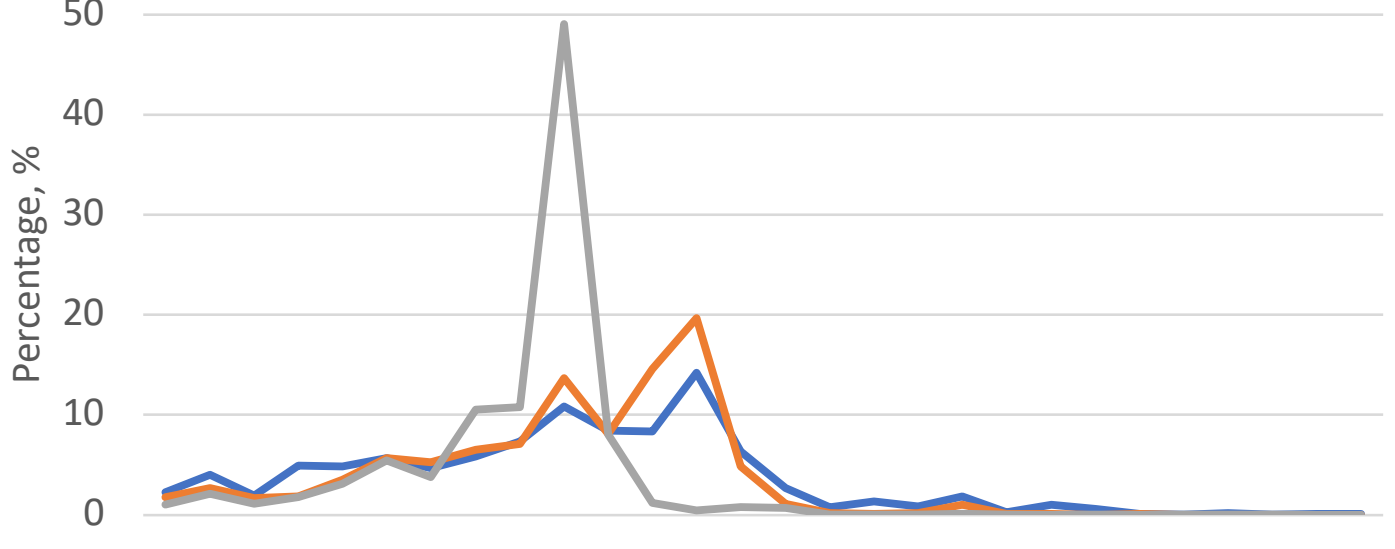

15161718192021222324252627282930313233343536373839404142 Read length

—IP ago2-1 IP AGO2-2

IP AGO 2-3

D
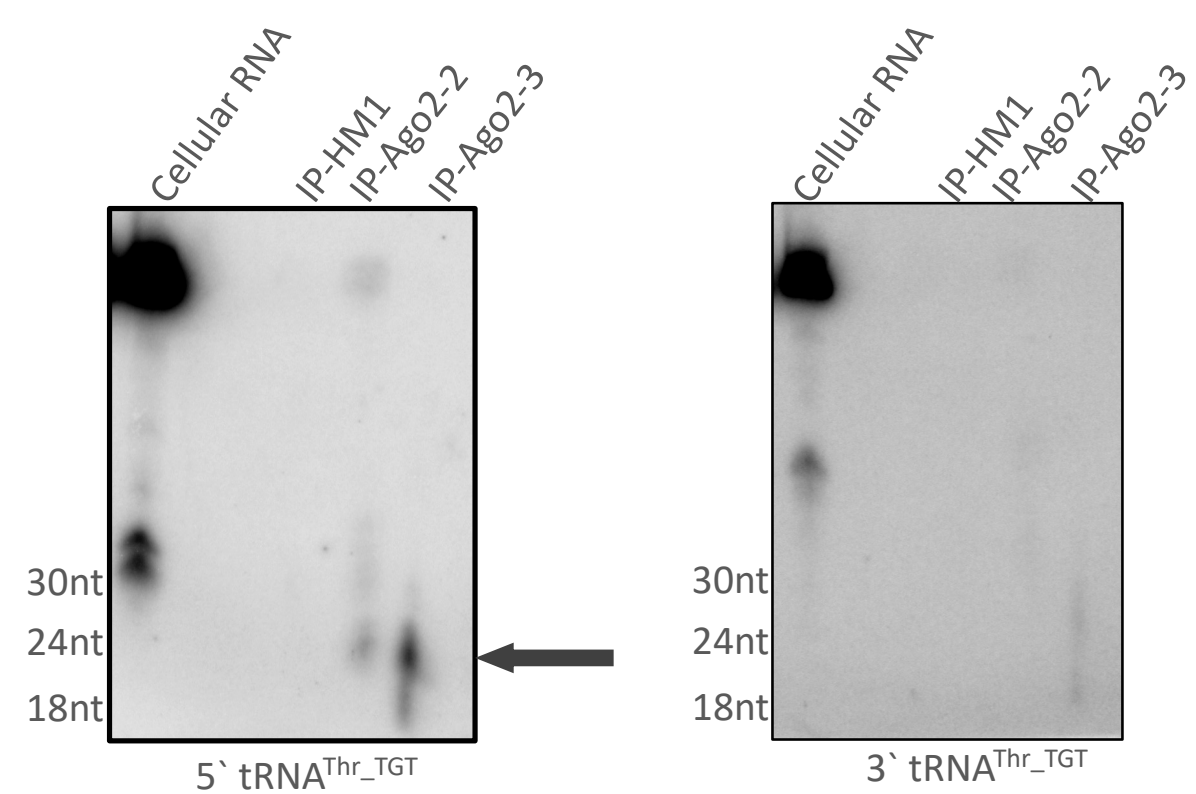

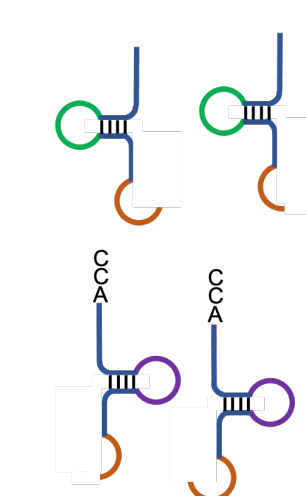

tRNA halves

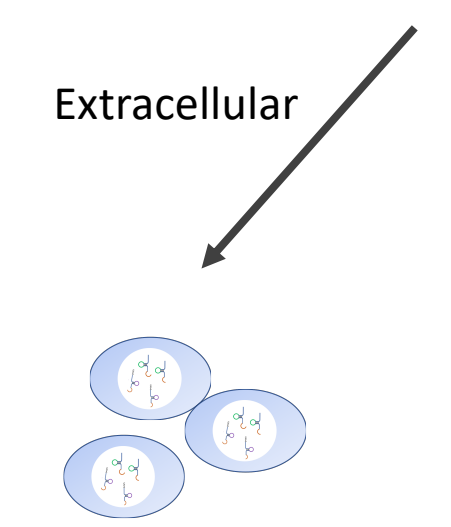

Extracellular vesicles

Extracellular Communication and signal to other parasites, host cells and other organisms

\section{Stress/encystation}

Angiogenin independent mechanism of cleavage

\section{.}

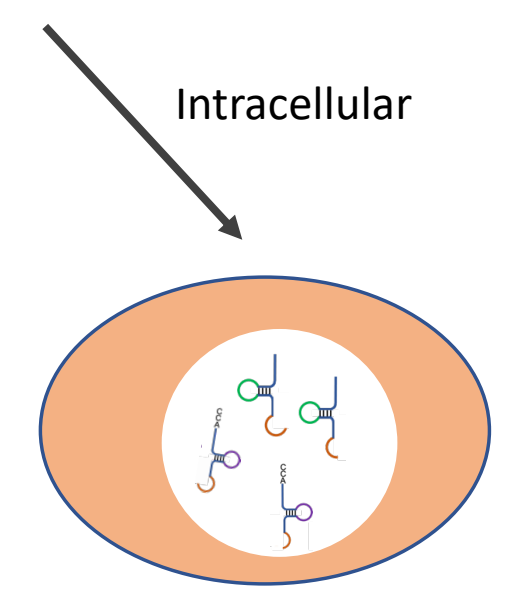

Modulation of protein translation?
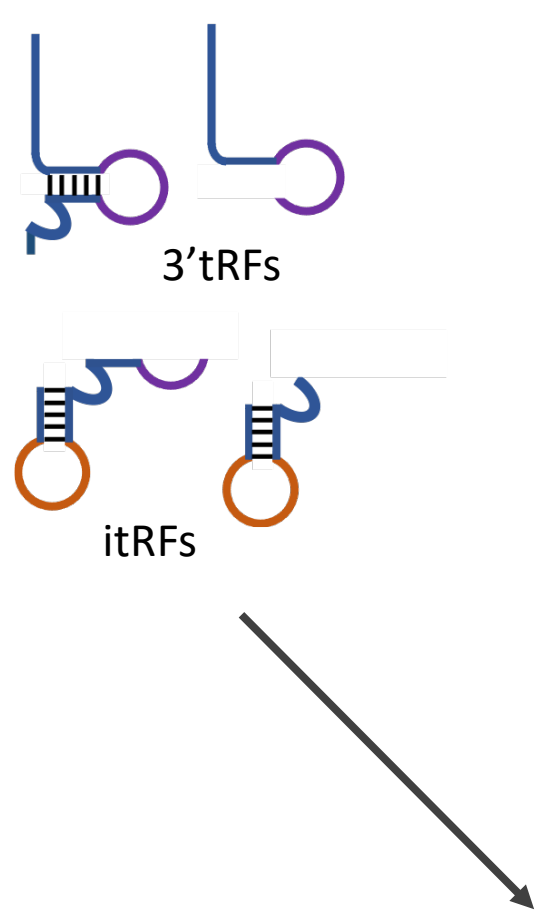

Other functions ?

Gene silencing 